\title{
Photodecomposition of Thienylsulfonyl Azides: Generation and Spectroscopic Characterization of Triplet Thienylsulfonyl Nitrenes and 3-Thienylnitrene
}

\author{
Yang Yang, ${ }^{\dagger}$ Guohai Deng, ${ }^{\dagger}$ Yan Lu, ${ }^{\dagger}$ Qian Liu, ${ }^{\dagger}$ Manabu Abe, ${ }^{\dagger}$ and Xiaoqing Zeng ${ }^{\dagger, *}$ \\ ${ }^{\dagger}$ College of Chemistry, Chemical Engineering and Materials Science, Soochow University, \\ Suzhou 215123, China. E-mail: xqzeng@suda.edu.cn \\ ${ }^{ \pm}$Department of Chemistry, Graduate School of Science, Hiroshima University, 1-3-1 Kagamiyama, \\ Higashi-Hiroshima Hiroshima 739-8526, Japan.
}

Table of Contents

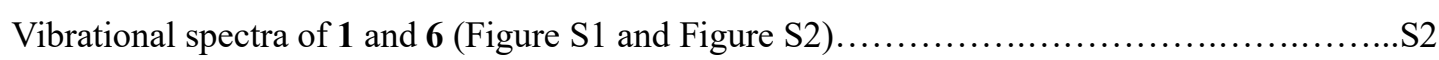

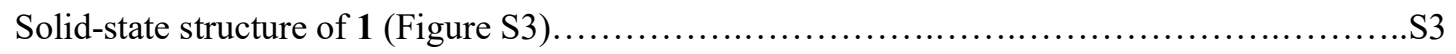

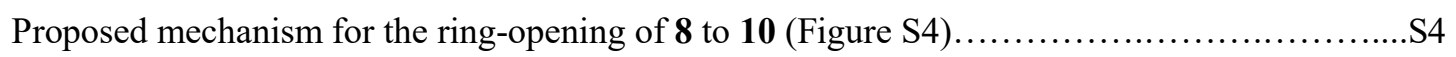

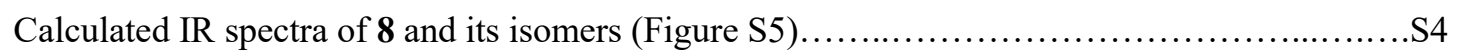

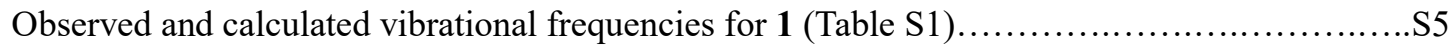

Observed and calculated vibrational frequencies for $\mathbf{6}$ (Table S2).............................. 6

Summary of crystal data and refinement results for $\mathbf{1}$ (Table S3) ................................. 7

Calculated and experimental structural parameters of $\mathbf{1}$ (Table S4)............................S7

TD-DFT calculated vertical transitions of nitrenes $\mathbf{2}, \mathbf{7}$, and $\mathbf{8}$ (Table S5) ........................S8

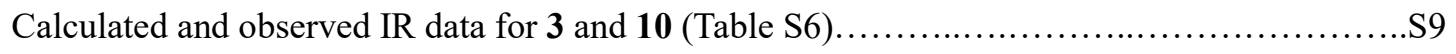

Observed band positions and calculated vibrational frequencies of 9 (Table S7) ...............S10

Calculated energies and atomic coordinates for all optimized structures......................S11 


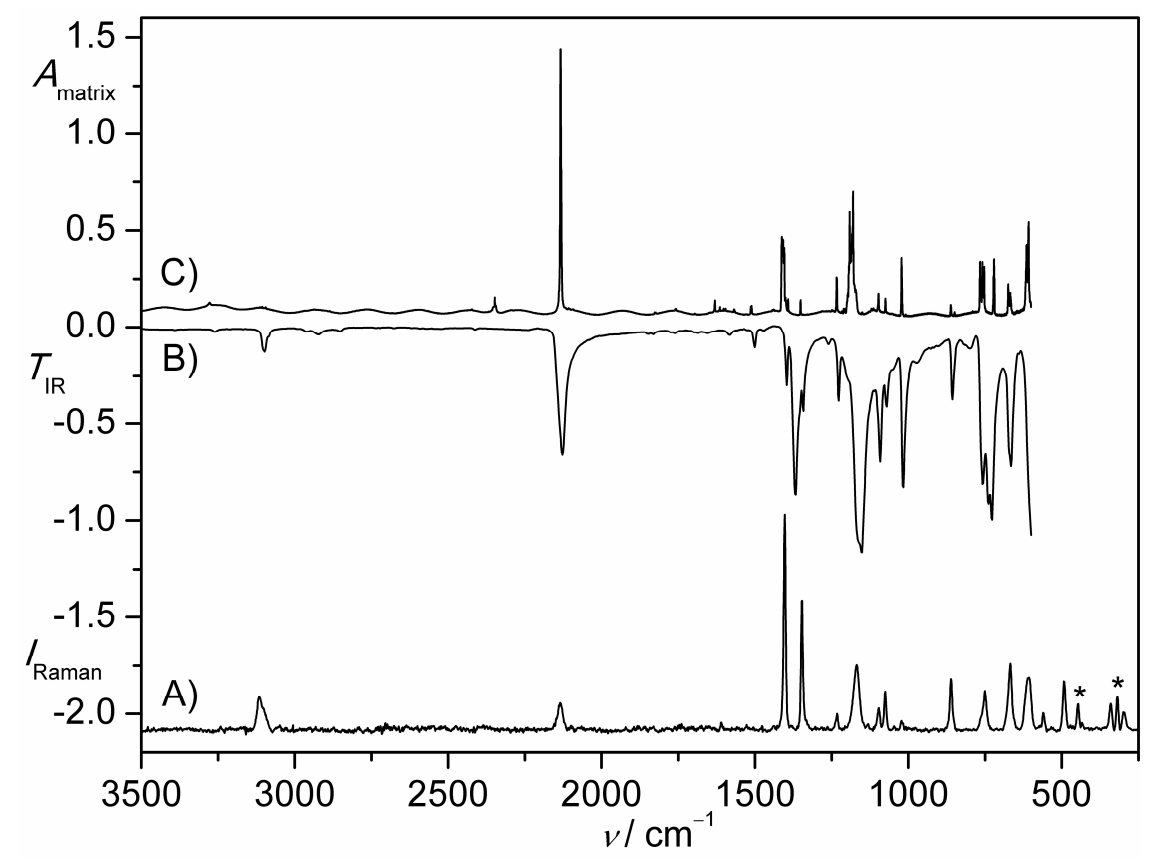

Figure S1. A) Raman spectrum of solid 2-thienylsulfonyl azide (1) in $\mathrm{CCl}_{4}$ at $298 \mathrm{~K}$, the bands of $\mathrm{CCl}_{4}$ are labeled with asterisks; B) IR spectrum of solid 1 at $298 \mathrm{~K}$; C) IR spectrum of $\mathbf{1}$ isolated in solid Ne-matrix at $2.8 \mathrm{~K}$.

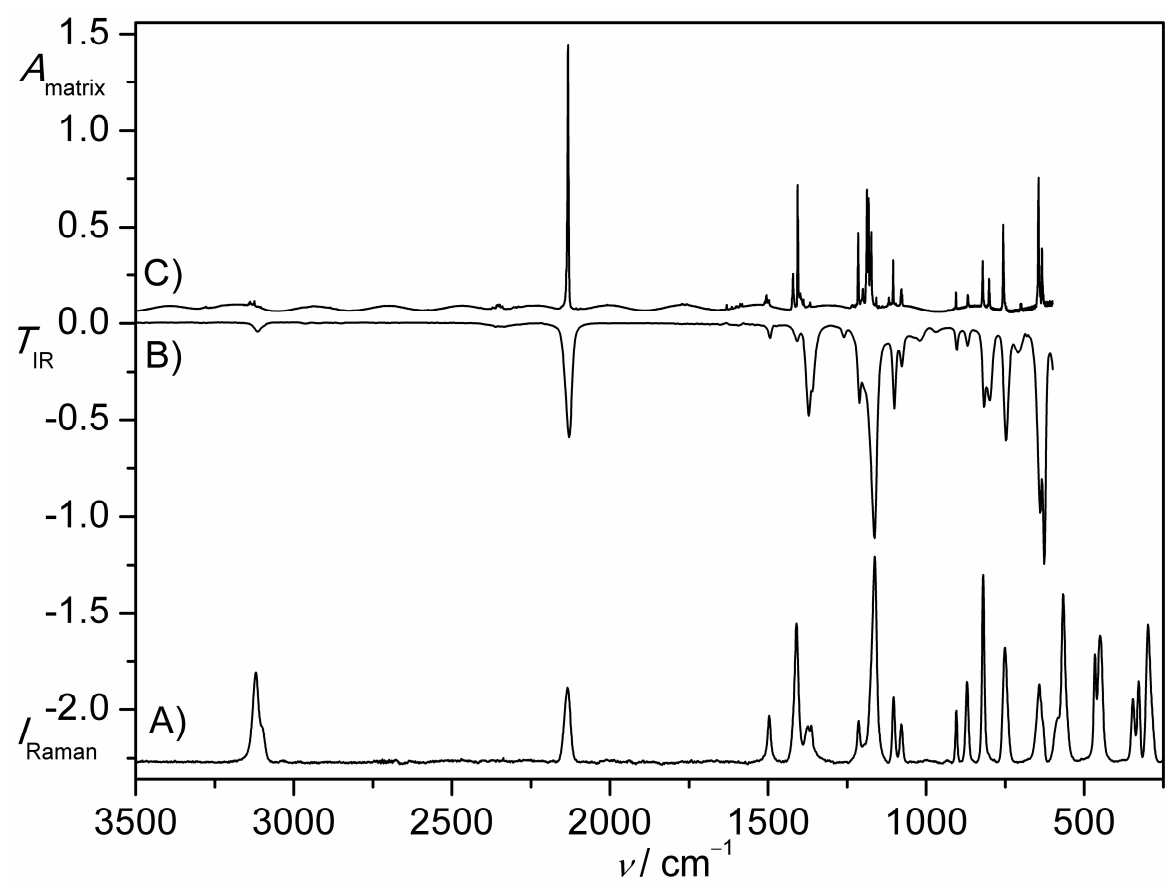

Figure S2. A) Raman spectrum of liquid 3-thienylsulfonyl azide (6) at $298 \mathrm{~K}$; B) IR spectrum of liquid 6 at 298 K; C) IR spectrum of 6 in solid Ne-matrix at $2.8 \mathrm{~K}$. 

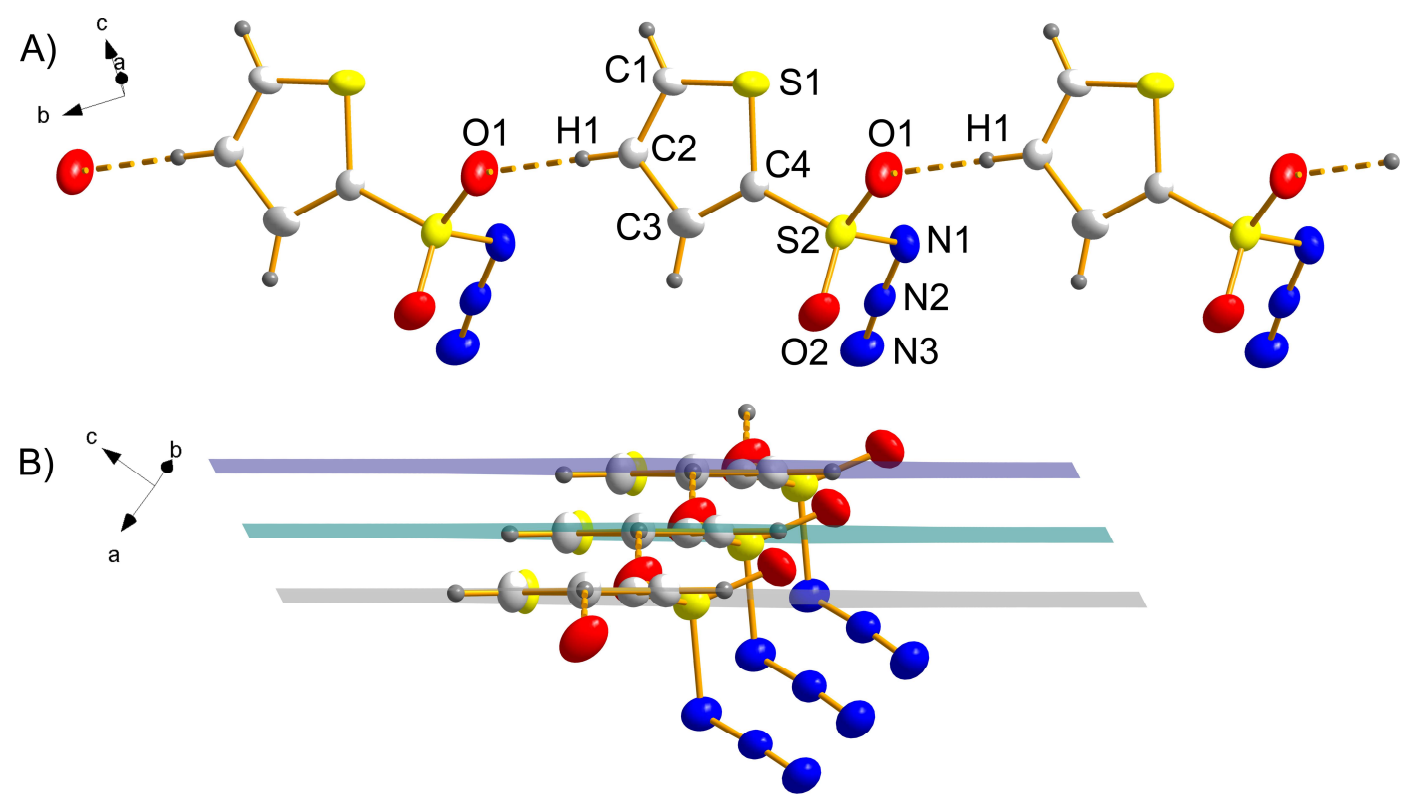

Figure S3. Solid-state structure of 1 with ellipsoids at 50\% probability. A): Top view; B) side view. Short intermolecular $\mathrm{H}^{\cdots} \mathrm{O}$ contacts $(2.518 \AA)$ are indicated by dash lines. The distance between two neighboring parallel planes is $0.947 \AA$. Selected structural parameters are $\mathrm{r}(\mathrm{S} 2-\mathrm{O} 1)=1.4141(19) \AA, \mathrm{r}(\mathrm{S} 2-\mathrm{O} 2)=1.4210(17) \AA, \mathrm{r}(\mathrm{S} 2-\mathrm{C} 4)$ $\left.=1.7164(19) \AA,(\mathrm{O} 1 \mathrm{~S} 2 \mathrm{O} 2)=121.55(12)^{\circ},(\mathrm{O} 1 \mathrm{~S} 2 \mathrm{~N} 1)=102.62(11)^{\circ},(\mathrm{O} 2 \mathrm{~S} 2 \mathrm{~N} 1)=108.75(10)^{\circ}\right)$. 


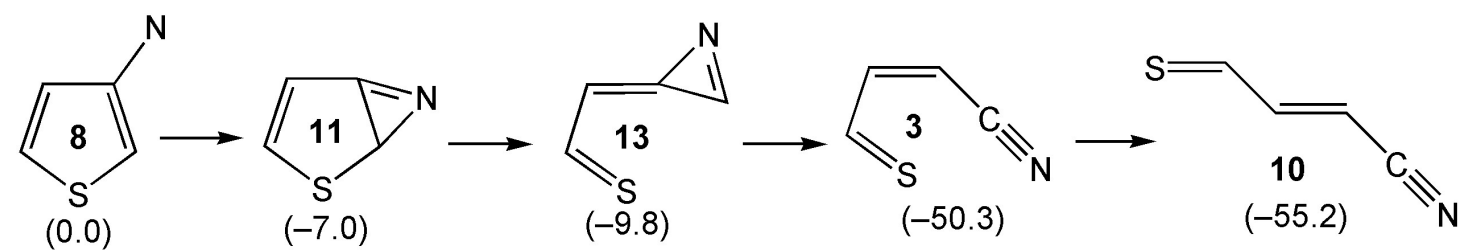

Figure S4. Proposed mechanism for the ring-opening of 3-thienylnitrene to (s-E)-4-thioxo-2-butenenitrile in the singlet state. The relative energies $\left(\mathrm{kcal} \mathrm{mol}^{-1}\right)$ calculated at the B3LYP/6-311++G(3df,3pd) level are given in parentheses.

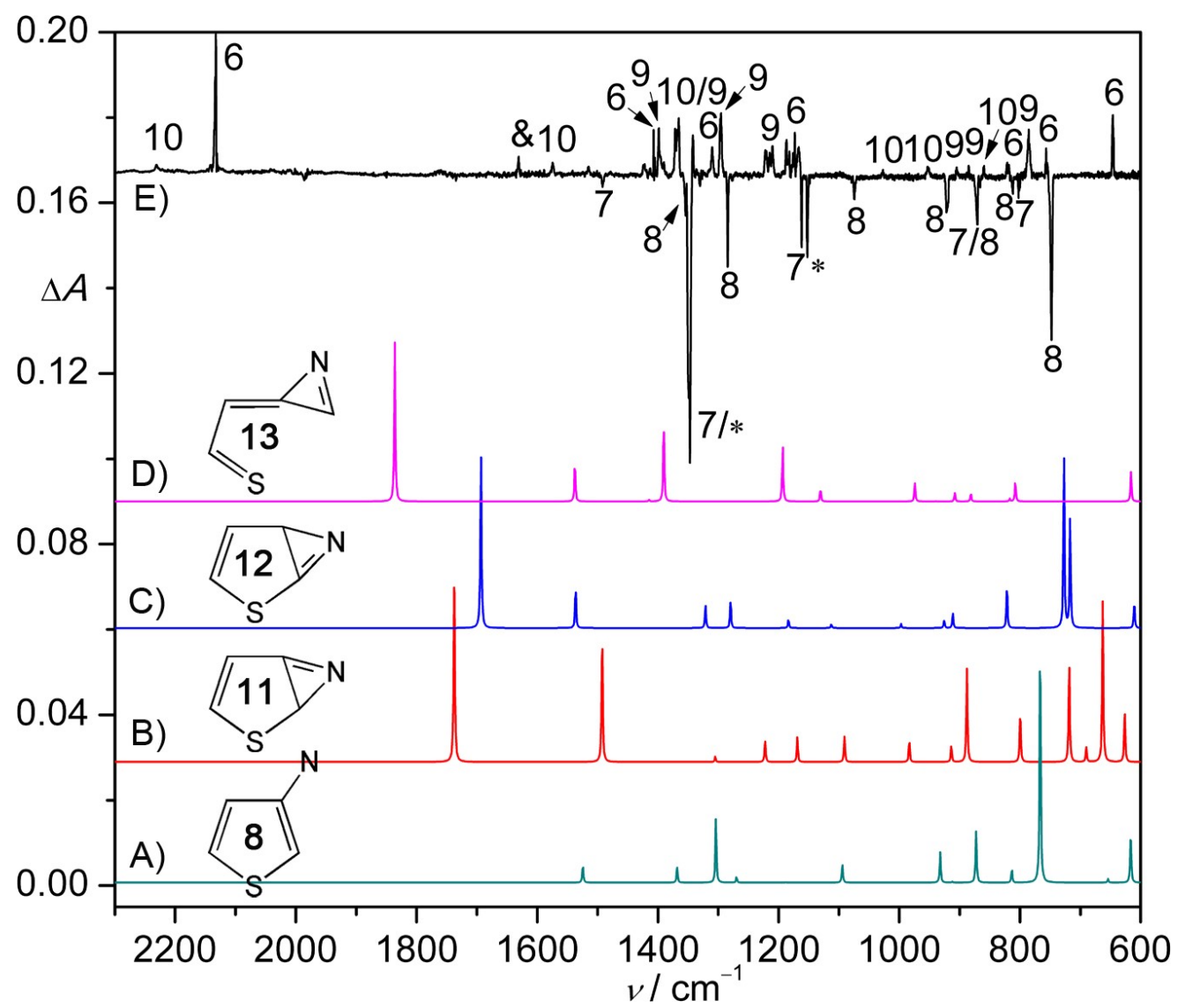

Figure S5. A) Calculated IR spectrum of triplet 8 at the B3LYP/6-311++G(3df,3pd) level; B, C) Calculated IR spectra of bicyclic azirines $\mathbf{1 1}$ and $\mathbf{1 2}$ at the B3LYP/6-311++G(3df,3pd) level; D) Calculated IR spectrum of azirine 13 at the B3LYP/6-311++G(3df,3pd) level. E) IR difference spectrum showing the change upon UV-light irradiation ( $365 \mathrm{~nm}, 65 \mathrm{~min}$ ) to 7 and 8 in Ne-marix at $2.8 \mathrm{~K}$, bands of $\mathrm{SO}_{2}\left({ }^{*}\right)$ and $\mathrm{H}_{2} \mathrm{O}(\&)$ are marked. 
Table S1. Observed and Calculated Vibrational Frequencies $\left(>500 \mathrm{~cm}^{-1}\right)$ for $\mathbf{1}$

\begin{tabular}{|c|c|c|c|c|c|c|}
\hline \multicolumn{5}{|l|}{ Observed $^{\mathrm{a}}$} & \multirow{2}{*}{$\begin{array}{l}\text { Calculated }^{\mathrm{b}} \\
\text { B3LYP } \\
{\left[C_{1}\right]} \\
\end{array}$} & \multirow[b]{2}{*}{$\begin{array}{l}\text { Approximate } \\
\text { assignment }^{\mathrm{c}}\end{array}$} \\
\hline $\begin{array}{l}\text { IR } \\
\left(\mathrm{N}_{2} \text {-matrix }\right)\end{array}$ & $\begin{array}{l}\text { IR } \\
\text { (Ne-matrix) }\end{array}$ & $\begin{array}{l}\text { IR } \\
\text { (Ar-matrix) }\end{array}$ & $\begin{array}{l}\text { IR } \\
\text { (solid) }\end{array}$ & $\begin{array}{l}\text { Raman } \\
\left(\text { in } \mathrm{CCl}_{4} \text { ) }\right.\end{array}$ & & \\
\hline & & & $3263 \mathrm{vw}$ & & $3249(<1)$ & $v(\mathrm{CH})$ \\
\hline & & & $3102 \mathrm{w}$ & $3116 w$ & $3234(2)$ & $v(\mathrm{CH})$ \\
\hline & & & $2926 \mathrm{vw}$ & & $3215(2)$ & $v(\mathrm{CH})$ \\
\hline 2136.4 vs & $2133.4 \mathrm{vs}$ & $2130.0 \mathrm{vs}$ & $2128 \mathrm{vs}$ & $2135 \mathrm{w}$ & $2253(530)$ & $v_{\text {antisym }}\left(\mathrm{N}_{3}\right)$ \\
\hline $1510.1 \mathrm{vw}$ & $1512.5 \mathrm{vw}$ & $1510.4 \mathrm{vw}$ & $1502 \mathrm{vw}$ & & $1541(9)$ & $v(\mathrm{C}=\mathrm{C})$ \\
\hline $1403.5 \mathrm{w}$ & $1412.6 \mathrm{w}$ & $1403.4 \mathrm{w}$ & $1396 \mathrm{w}$ & 1402 vs & $1434(21)$ & $v(\mathrm{C}-\mathrm{C})+\rho(\mathrm{CH})$ \\
\hline $1398.5 \mathrm{~s}$ & $1409.4 \mathrm{~s}$ & $1401.3 \mathrm{~s}$ & $1369 \mathrm{w}$ & $1362 \mathrm{vw}$ & 1409 (169) & $v_{\text {antisym }}\left(\mathrm{SO}_{2}\right)$ \\
\hline $1350.1 \mathrm{vw}$ & $1351.7 \mathrm{vw}$ & $1350.5 \mathrm{vw}$ & $1344 \mathrm{vw}$ & $1347 \mathrm{~s}$ & $1373(9)$ & $v(\mathrm{C}=\mathrm{C})$ \\
\hline $1234.4 \mathrm{~s}$ & $1233.6 \mathrm{~s}$ & $1231.1 \mathrm{~m}$ & $1227 \mathrm{~m}$ & $1232 \mathrm{w}$ & $1268(239)$ & $v_{\text {sym }}\left(\mathrm{N}_{3}\right)$ \\
\hline $1210.2 \mathrm{vw}$ & $1210.3 \mathrm{vw}$ & $1205.8 \mathrm{vw}$ & & & $1258(29)$ & $\rho(\mathrm{CH})$ \\
\hline $1180.5 \mathrm{~s}$ & $1180.5 \mathrm{~s}$ & $1179.8 \mathrm{~s}$ & $1152 \mathrm{~s}$ & $1168 \mathrm{~s}$ & $1185(214)$ & $v_{\mathrm{sym}}\left(\mathrm{SO}_{2}\right)$ \\
\hline $1097.4 \mathrm{vw}$ & $1097.6 \mathrm{vw}$ & $1096.4 \mathrm{vw}$ & $1093 \mathrm{w}$ & $1096 \mathrm{w}$ & $1113(7)$ & $\rho(\mathrm{CH})$ \\
\hline $1075.2 \mathrm{vw}$ & $1075.0 \mathrm{vw}$ & $1073.7 \mathrm{vw}$ & $1072 \mathrm{w}$ & $1075 \mathrm{w}$ & $1088(4)$ & $\rho(\mathrm{CH})+v(\mathrm{CS})$ \\
\hline \multirow[t]{3}{*}{$1024.1 \mathrm{~m}$} & $1021.9 \mathrm{~m}$ & $1020.8 \mathrm{~m}$ & $1016 \mathrm{~m}$ & & $1024(64)$ & $\rho(\mathrm{CH})+v\left(\mathrm{CS}_{\text {ring }}\right)$ \\
\hline & & & & & $949(<1)$ & $\tau(\mathrm{CH})$ \\
\hline & & & & & $877(2)$ & $\omega(\mathrm{CH})$ \\
\hline \multirow[t]{2}{*}{$864.3 \mathrm{vw}$} & $861.9 \mathrm{vw}$ & $859.9 \mathrm{vw}$ & $856 \mathrm{w}$ & $861 \mathrm{~s}$ & $868(8)$ & ring distortion \\
\hline & & & & & $758(3)$ & ring distortion \\
\hline $751.7 \mathrm{~m}$ & $752.6 \mathrm{~m}$ & $750.9 \mathrm{~m}$ & $758 \mathrm{~m}$ & $750 \mathrm{~m}$ & $740(108)$ & $\omega(\mathrm{SN})+\delta_{\text {i.p. }}\left(\mathrm{N}_{3}\right)$ \\
\hline $732.1 \mathrm{~m}$ & $721.0 \mathrm{~m}$ & $719.7 \mathrm{~m}$ & $728 \mathrm{~m}$ & & $734(37)$ & $\omega(\mathrm{CH})$ \\
\hline $672.3 \mathrm{~m}$ & $674.7 \mathrm{~m}$ & $669.9 \mathrm{~m}$ & $665 \mathrm{~m}$ & $668 \mathrm{~m}$ & $675(70)$ & ring breathing $+\delta\left(\mathrm{SO}_{2}\right)$ \\
\hline \multirow[t]{4}{*}{$607.4 \mathrm{~s}$} & $608.2 \mathrm{~s}$ & $609.5 \mathrm{~s}$ & $600 \mathrm{~s}$ & $608 \mathrm{~m}$ & $610(213)$ & $v(\mathrm{SN})+\delta\left(\mathrm{SO}_{2}\right)$ \\
\hline & & & & & $584(13)$ & $\delta_{\text {o.o.p. }}\left(\mathrm{N}_{3}\right)$ \\
\hline & & & & $560 \mathrm{w}$ & $577(58)$ & $\omega(\mathrm{CH})$ \\
\hline & & & & & $566(50)$ & $\omega(\mathrm{CH})+\delta\left(\mathrm{SO}_{2}\right)$ \\
\hline
\end{tabular}

\footnotetext{
${ }^{a}$ Band positions and relative intensities: $\mathrm{vs}=$ very strong; $\mathrm{s}=$ strong; $\mathrm{m}=$ medium strong; $\mathrm{w}=$ weak; $\mathrm{vw}=$ very weak. ${ }^{b}$ Calculated with the $6-311++\mathrm{G}(3 \mathrm{df}, 3 \mathrm{pd})$ basis set, molecular symmetry in square bracket and IR intensities $\left(\mathrm{km} \mathrm{mol}^{-1}\right)$ in parentheses. ${ }^{c}$ Tentative assignment based on the calculated vibrational displacement vectors, $v=$ stretching; $\rho$ = rocking; $\tau=$ twisting; $\omega=$ wagging; $\delta=$ bending.
} 
Table S2. Observed and Calculated Vibrational Frequencies $\left(>500 \mathrm{~cm}^{-1}\right)$ for $\mathbf{6}$

\begin{tabular}{|c|c|c|c|c|c|c|}
\hline \multicolumn{5}{|l|}{ Observed $^{\mathrm{a}}$} & \multirow{2}{*}{$\begin{array}{l}\text { Calculated }^{\mathrm{b}} \\
\text { B3LYP } \\
{\left[C_{1}\right]} \\
\end{array}$} & \multirow[b]{2}{*}{$\begin{array}{l}\text { Approximate } \\
\text { assignment }^{\mathrm{c}}\end{array}$} \\
\hline $\begin{array}{l}\mathrm{IR} \\
\left(\mathrm{N}_{2} \text {-matrix }\right)\end{array}$ & $\begin{array}{l}\text { IR } \\
\text { (Ne-matrix) }\end{array}$ & $\begin{array}{l}\text { IR } \\
\text { (Ar-matrix) }\end{array}$ & $\begin{array}{l}\text { IR } \\
\text { (liquid) }\end{array}$ & $\begin{array}{l}\text { Raman } \\
\text { (liquid) }\end{array}$ & & \\
\hline $3128.4 \mathrm{vw}$ & $3124.2 \mathrm{vw}$ & $3124.8 \mathrm{vw}$ & $3114 \mathrm{w}$ & $3119 \mathrm{~m}$ & $\begin{array}{l}3267(11) \\
3256(1) \\
3231(2)\end{array}$ & $\begin{array}{l}v(\mathrm{CH}) \\
v(\mathrm{CH}) \\
v(\mathrm{CH})\end{array}$ \\
\hline 2134.3 vs & 2132.6 vs & 2130.1 vs & $2129 \mathrm{vs}$ & $2133 \mathrm{~m}$ & 2253 (575) & $v_{\text {antisym }}\left(\mathrm{N}_{3}\right)$ \\
\hline $1499.5 \mathrm{w}$ & $1505.6 \mathrm{w}$ & $1501.1 \mathrm{w}$ & $1492 \mathrm{w}$ & $1497 \mathrm{~m}$ & $1531(12)$ & $v(\mathrm{C}=\mathrm{C})$ \\
\hline $1409.3 \mathrm{w}$ & $1421.3 \mathrm{w}$ & $1419.8 \mathrm{w}$ & $1408 \mathrm{w}$ & $1410 \mathrm{~s}$ & $1437(32)$ & $v(\mathrm{C}=\mathrm{C})$ \\
\hline $1393.3 \mathrm{~s}$ & $1406.7 \mathrm{~s}$ & $1399.9 \mathrm{~s}$ & $1371 \mathrm{~s}$ & $1373 \mathrm{w}$ & $1406(138)$ & $v_{\text {antisym }}\left(\mathrm{SO}_{2}\right)$ \\
\hline $1366.4 \mathrm{vw}$ & $1367.0 \mathrm{vw}$ & $1366.7 \mathrm{vw}$ & $1361 \mathrm{w}$ & $1364 \mathrm{w}$ & $1391(23)$ & $\rho(\mathrm{CH})$ \\
\hline $1215.3 \mathrm{~s}$ & $1215.1 \mathrm{~s}$ & $1212.8 \mathrm{~s}$ & $1211 \mathrm{~m}$ & $1214 \mathrm{w}$ & $1271(253)$ & $v_{\text {sym }}\left(\mathrm{N}_{3}\right)$ \\
\hline $1203.0 \mathrm{w}$ & $1200.1 \mathrm{w}$ & $1203.2 \mathrm{w}$ & & & $1235(24)$ & $\rho(\mathrm{CH})$ \\
\hline $1180.5 \mathrm{~s}$ & $1187.2 \mathrm{~s}$ & $1174.4 \mathrm{~s}$ & 1164 vs & 1162 vs & $1186(205)$ & $v_{\text {sym }}\left(\mathrm{SO}_{2}\right)$ \\
\hline $1106.5 \mathrm{~m}$ & $1104.2 \mathrm{~m}$ & $1103.1 \mathrm{~m}$ & $1100 \mathrm{~m}$ & $1103 \mathrm{~m}$ & $1120(25)$ & $\rho(\mathrm{CH})$ \\
\hline $1078.8 \mathrm{w}$ & $1078.1 \mathrm{w}$ & $1077.2 \mathrm{w}$ & $1077 \mathrm{w}$ & $1078 \mathrm{w}$ & $\begin{array}{l}1098(24) \\
920(<1)\end{array}$ & $\begin{array}{l}\rho(\mathrm{CH}) \\
\tau(\mathrm{CH})\end{array}$ \\
\hline $904.8 \mathrm{vw}$ & $905.7 \mathrm{vw}$ & $904.9 \mathrm{vw}$ & $903 \mathrm{vw}$ & $904 \mathrm{~m}$ & $912(6)$ & ring distortion \\
\hline $872.4 \mathrm{vw}$ & $868.5 \mathrm{vw}$ & $866.3 \mathrm{vw}$ & $869 \mathrm{vw}$ & $871 \mathrm{~s}$ & $875(11)$ & $v\left(\mathrm{CS}_{\text {ring }}\right)$ \\
\hline $817.9 \mathrm{~m}$ & $820.9 \mathrm{~m}$ & $818.3 \mathrm{~m}$ & $817 \mathrm{~m}$ & 820 vs & $826(30)$ & $\omega(\mathrm{CH})$ \\
\hline $804.5 \mathrm{~m}$ & $801.4 \mathrm{~m}$ & $799.6 \mathrm{~m}$ & $800 \mathrm{~m}$ & & $822(28)$ & $v\left(\mathrm{CS}_{\text {ring }}\right)$ \\
\hline $756.3 \mathrm{~s}$ & $756.3 \mathrm{~s}$ & $753.2 \mathrm{~s}$ & $747 \mathrm{~s}$ & $751 \mathrm{~m}$ & $734(76)$ & $v(\mathrm{SN})+\delta_{\text {i.p. }}\left(\mathrm{N}_{3}\right)$ \\
\hline $707.4 \mathrm{vw}$ & $699.1 \mathrm{vw}$ & $699.6 \mathrm{w}$ & $708 \mathrm{w}$ & & $715(11)$ & $\omega(\mathrm{CH})$ \\
\hline $643.4 \mathrm{~s}$ & $644.5 \mathrm{~s}$ & $642.0 \mathrm{~s}$ & $639 \mathrm{~s}$ & $642 \mathrm{~m}$ & $651(144)$ & ring distortion \\
\hline $632.9 \mathrm{~s}$ & $633.8 \mathrm{~s}$ & $632.2 \mathrm{~s}$ & $626 \mathrm{vs}$ & $\begin{array}{l}636 \mathrm{w} \\
586 \mathrm{w} \\
566 \mathrm{~s}\end{array}$ & $\begin{array}{l}640(37) \\
592(171) \\
584(15) \\
561(60)\end{array}$ & $\begin{array}{c}\text { ring breathing } \\
\delta_{\text {o.o.p. }}\left(\mathrm{N}_{3}\right)+\delta\left(\mathrm{SO}_{2}\right) \\
\delta_{\text {o.o.p. }}\left(\mathrm{N}_{3}\right) \\
\delta\left(\mathrm{SO}_{2}\right)+\omega(\mathrm{CH})\end{array}$ \\
\hline
\end{tabular}

${ }^{a}$ Band positions and relative intensities: vs = very strong; $\mathrm{s}=$ strong; $\mathrm{m}=$ medium strong; $\mathrm{w}=$ weak; $\mathrm{vw}=$ very weak. ${ }^{b}$ Calculated with the $6-311++\mathrm{G}(3 \mathrm{df}, 3 \mathrm{pd})$ basis set, molecular symmetry in square bracket and IR intensities $\left(\mathrm{km} \mathrm{mol}^{-1}\right)$ in parentheses. ${ }^{c}$ Tentative assignment based on the calculated vibrational displacement vectors, $v=$ stretching; $\rho$ = rocking; $\tau=$ twisting; $\omega=$ wagging; $\delta=$ bending. 
Table S3. Summary of Crystal Data and Refinement Results for 1

\begin{tabular}{|c|c|}
\hline Chemical formula & $\mathrm{C}_{4} \mathrm{H}_{3} \mathrm{~N}_{3} \mathrm{O}_{2} \mathrm{~S}_{2}$ \\
\hline space group & orthorhombic $P 2{ }_{1} 2_{1} 2_{1}$ \\
\hline$a(\AA)$ & $5.0271(2)$ \\
\hline$b(\AA)$ & $6.5077(3)$ \\
\hline$c(\AA)$ & $22.1546(9)$ \\
\hline$\alpha(\mathrm{deg})$ & 90 \\
\hline$\beta(\operatorname{deg})$ & 90 \\
\hline$\gamma(\operatorname{deg})$ & 90 \\
\hline$V\left(\AA^{3}\right)$ & $724.78(5)$ \\
\hline$Z$ (molecules/unit cell) & 4 \\
\hline mol wt & 189.21 \\
\hline calculated density (g.cm $\left.{ }^{-3}\right)$ & 1.734 \\
\hline $\mathrm{T}(\mathrm{K})$ & $120(2)$ \\
\hline$\mu\left(\mathrm{mm}^{-1}\right)$ & 0.682 \\
\hline$R_{1}^{\mathrm{a}}$ & 0.0223 \\
\hline$w R_{2}^{\mathrm{b}}$ & 0.0573 \\
\hline
\end{tabular}

Table S4. Calculated and Experimental Structural Parameters of 1

\begin{tabular}{|c|c|c|c|c|c|}
\hline Parameters $^{\mathrm{a}}$ & B3LYP $^{b}$ & MPW1PW91 $^{b}$ & ${\mathrm{BP} 86^{b}}^{\mathrm{b}}$ & M06-2X & $\mathrm{XRD}^{\mathrm{c}}$ \\
\hline$r(\mathrm{~S} 2-\mathrm{O} 1)$ & 1.425 & 1.418 & 1.440 & 1.417 & $1.4141(19)$ \\
\hline$r(\mathrm{~S} 2-\mathrm{O} 2)$ & 1.432 & 1.426 & 1.448 & 1.425 & $1.4210(17)$ \\
\hline$r(\mathrm{~S} 2-\mathrm{C} 4)$ & 1.743 & 1.732 & 1.750 & 1.738 & $1.7164(19)$ \\
\hline$r(\mathrm{~S} 2-\mathrm{N} 1)$ & 1.724 & 1.701 & 1.755 & 1.699 & $1.6848(18)$ \\
\hline$r(\mathrm{~N} 1-\mathrm{N} 2)$ & 1.237 & 1.232 & 1.242 & 1.241 & $1.253(2)$ \\
\hline$r(\mathrm{~N} 2-\mathrm{N} 3)$ & 1.122 & 1.117 & 1.140 & 1.111 & $1.106(3)$ \\
\hline$\angle(\mathrm{O} 1 \mathrm{~S} 2 \mathrm{O} 2)$ & 123.0 & 123.0 & 123.5 & 123.1 & $121.55(12)$ \\
\hline$\angle(\mathrm{O} 1 \mathrm{~S} 2 \mathrm{~N} 1)$ & 108.2 & 103.2 & 102.4 & 103.6 & $102.62(11)$ \\
\hline$\angle(\mathrm{O} 2 \mathrm{~S} 2 \mathrm{~N} 1)$ & 103.4 & 108.2 & 108.3 & 108.1 & $108.75(10)$ \\
\hline$\angle(\mathrm{C} 4 \mathrm{~S} 2 \mathrm{~N} 1)$ & 103.5 & 103.4 & 103.1 & 103.0 & $104.13(10)$ \\
\hline$\angle(\mathrm{S} 2 \mathrm{~N} 1 \mathrm{~N} 2)$ & 115.1 & 114.8 & 114.7 & 112.4 & $111.32(14)$ \\
\hline$\angle(\mathrm{N} 1 \mathrm{~N} 2 \mathrm{~N} 3)$ & 174.7 & 174.9 & 174.4 & 176.2 & $175.4(2)$ \\
\hline$\varphi(\mathrm{O} 1 \mathrm{~S} 2-\mathrm{N} 1 \mathrm{~N} 2)$ & 163.7 & 165.6 & 165.8 & 169.6 & $173.2(1)$ \\
\hline$\varphi(\mathrm{O} 2 \mathrm{~S} 2-\mathrm{N} 1 \mathrm{~N} 2)$ & 32.0 & 33.8 & 33.9 & 37.5 & $43.2(2)$ \\
\hline$\varphi(\mathrm{C} 4 \mathrm{~S} 2-\mathrm{N} 1 \mathrm{~N} 2)$ & -82.5 & -80.5 & -80.6 & -76.3 & $-72.6(2)$ \\
\hline$\varphi(\mathrm{S} 2 \mathrm{~N} 1-\mathrm{N} 2 \mathrm{~N} 3)$ & -178.5 & -179.3 & -178.8 & 176.9 & $177.1(3)$ \\
\hline
\end{tabular}


Table S5. Calculated Vertical Transitions for 2, 7, and 8 at the TD-B3LYP/6-311++G(3df,3pd)

Level.

\begin{tabular}{|c|c|c|c|c|c|c|c|c|c|c|c|}
\hline $\begin{array}{l}\mathbf{2} \\
\text { singlet }\end{array}$ & & triplet & & $\begin{array}{l}7 \\
\text { singlet }\end{array}$ & & triplet & & $\begin{array}{l}\mathbf{8} \\
\text { singlet }\end{array}$ & & triplet & \\
\hline $\begin{array}{l}\text { energy } \\
\text { (nm) }\end{array}$ & $\begin{array}{l}\text { oscillator } \\
\text { strength }\end{array}$ & $\begin{array}{l}\text { energy } \\
(\mathrm{nm})\end{array}$ & $\begin{array}{l}\text { oscillator } \\
\text { strength }\end{array}$ & $\begin{array}{l}\text { energy } \\
(\mathrm{nm})\end{array}$ & $\begin{array}{l}\text { oscillator } \\
\text { strength }\end{array}$ & $\begin{array}{l}\text { energy } \\
\text { (nm) }\end{array}$ & $\begin{array}{l}\text { oscillator } \\
\text { strength }\end{array}$ & $\begin{array}{l}\text { energy } \\
(\mathrm{nm})\end{array}$ & $\begin{array}{l}\text { oscillator } \\
\text { strength }\end{array}$ & $\begin{array}{l}\text { energy } \\
(\mathrm{nm})\end{array}$ & $\begin{array}{l}\text { oscillator } \\
\text { strength }\end{array}$ \\
\hline 528 & 0.0040 & 526 & 0.0096 & 520 & 0.0031 & 522 & 0.0042 & 370 & 0.0021 & 413 & 0.0006 \\
\hline 301 & 0.0122 & 479 & 0.0110 & 302 & 0.0045 & 446 & 0.0002 & 336 & 0.0133 & 334 & 0.0206 \\
\hline 299 & 0.0676 & 468 & 0.0498 & 287 & 0.0231 & 423 & 0.0614 & 308 & 0.0019 & 299 & 0.0019 \\
\hline 290 & 0.0029 & 396 & 0.0023 & 276 & 0.0304 & 390 & 0.0002 & 274 & 0.0026 & 296 & 0.0073 \\
\hline 246 & 0.0032 & 347 & 0.0005 & 246 & 0.0023 & 352 & 0.0008 & 262 & 0.0089 & 280 & 0.0046 \\
\hline 234 & 0.0683 & 334 & 0.0273 & 235 & 0.0246 & 334 & 0.0051 & 250 & 0.0863 & 278 & 0.0866 \\
\hline 227 & 0.1156 & 327 & 0.0091 & 223 & 0.0093 & 331 & 0.0051 & 246 & 0.0851 & 274 & 0.0009 \\
\hline 223 & 0.0095 & 308 & 0.0004 & 215 & 0.0743 & 312 & 0.0003 & 231 & 00107 & 238 & 0.0013 \\
\hline
\end{tabular}


Table S6. Calculated (B3LYP/6-311++G(3df,3pd)) and Observed IR Data for the Conformers of 4-Thioxo-2-butenenitrile at the B3LYP/6-311++G(3df,3pd) Level.

\begin{tabular}{|c|c|c|c|c|c|c|c|c|c|c|c|}
\hline \multicolumn{6}{|l|}{ Calculated } & syn- & & \multicolumn{4}{|c|}{ Observed $^{\mathrm{d}}$} \\
\hline-607.193093 & & $-607.192095^{\mathrm{a}}$ & & -607.189872 & & -607.185206 & & & & & \\
\hline$v^{b}$ & $\Delta v^{\mathrm{c}}$ & $v^{\mathrm{b}}$ & $\Delta v^{\mathrm{c}}$ & $v^{b}$ & $\Delta v^{\mathrm{c}}$ & $v^{b}$ & $\Delta v^{\mathrm{c}}$ & $v$ & $\Delta v$ & $\bar{v}$ & $\Delta v$ \\
\hline $3190(1)$ & 0 & $3193(<1)$ & 0 & $3174(3)$ & 0 & 3175 (2) & 0 & & & & \\
\hline $3166(<1)$ & 0 & $3176(4)$ & 0 & $3164(4)$ & 0.7 & $3157(8)$ & 0 & & & & \\
\hline $3086(11)$ & 0.2 & $3106(3)$ & 0 & $3101(14)$ & 0.3 & $3088(21)$ & 0 & & & & \\
\hline $2322(6)$ & 29.0 & $2321(6)$ & 29.2 & $2319(5)$ & 30.0 & $2311(<1)$ & 28.9 & 2232.5 & 27.3 & 2230.9 & 26.3 \\
\hline $1636(21)$ & 0 & $1620(14)$ & 0 & $1648(24)$ & 1.1 & $1639(24)$ & 0 & 1571.3 & $<0.5$ & 1574.4 & $<0.5$ \\
\hline $1395(50)$ & 0 & 1439 (19) & 0.4 & $1398(26)$ & 0 & $1440(3)$ & 0.4 & & & 1371.1 & $<0.5$ \\
\hline $1319(4)$ & 0 & $1343(12)$ & 0 & $1340(1)$ & 0.3 & $1396(16)$ & 0 & 1332.7 & $<0.5$ & & \\
\hline $1253(2)$ & 0 & $1240(9)$ & 0.3 & $1273(39)$ & 0.1 & $1275(8)$ & 0.1 & 1306.5 & $<0.5$ & & \\
\hline $1186(60)$ & 0 & $1157(34)$ & 0.3 & $1163(57)$ & 0.9 & $1153(22)$ & 0.7 & 1117.9 & $<0.5$ & 1166.6 & $<0.5$ \\
\hline $1069(4)$ & 1.6 & $1057(20)$ & 1.0 & $1038(3)$ & 6.2 & $1024(<1)$ & 0 & & & & \\
\hline $1013(37)$ & 0 & $1022(<1)$ & 0 & $1016(17)$ & 0 & $959(8)$ & 0.5 & & & 1027.0 & $<0.5$ \\
\hline 989 (15) & 5.4 & $922(8)$ & 0 & $943(21)$ & 0 & $910(<1)$ & 0 & & & 953.1 & 3.7 \\
\hline 937 (18) & 0.1 & $911(4)$ & 5.9 & 899 (2) & 0 & $872(7)$ & 0.2 & 859.9 & $<0.5$ & 859.5 & $<0.5$ \\
\hline $847(<1)$ & 0.8 & $802(36)$ & 0.1 & $828(11)$ & 0 & $759(36)$ & 0.2 & 764.5 & $<0.5$ & & \\
\hline $580(2)$ & 1.8 & $687(<1)$ & 1.8 & $625(16)$ & 2.9 & $757(6)$ & 3.2 & 749.2 & $<0.5$ & & \\
\hline $500(3)$ & 2.0 & $519(2)$ & 1.2 & $511(3)$ & 1.1 & $506(5)$ & 0.7 & & & & \\
\hline
\end{tabular}

${ }^{a}$ Calculated total energies (Hartree). ${ }^{b}$ Calculated harmonic frequencies $\left(\mathrm{cm}^{-1}\right.$, unscaled) and infrared intensities $\left(\mathrm{km} \mathrm{mol}^{-1}\right)$ in parentheses. ${ }^{c}$ Calculated ${ }^{15} \mathrm{~N}$ isotopic shifts $\left(\mathrm{cm}^{-1}\right)$. ${ }^{\mathrm{d}}$ Observed data $\left(\mathrm{cm}^{-1}\right)$ for the most intense bands in Ne-matrix at $2.8 \mathrm{~K}$. 
Table S7. Observed Band Positions and Calculated Vibrational Frequencies $\left(>500 \mathrm{~cm}^{-1}\right)$ of 9

\begin{tabular}{|c|c|c|c|c|c|c|c|}
\hline \multirow{2}{*}{\multicolumn{2}{|c|}{$\begin{array}{l}\mathbf{5} \\
\text { Calculated }^{\mathrm{a}}\end{array}$}} & \multicolumn{5}{|l|}{9} & \multirow{3}{*}{$\begin{array}{l}\text { Tentative } \\
\text { assignment }^{\mathrm{c}}\end{array}$} \\
\hline & & \multirow{2}{*}{\multicolumn{2}{|c|}{$\begin{array}{l}\text { Calculated }^{\text {a }} \\
\text { B3LYP }\end{array}$}} & \multicolumn{3}{|l|}{ Observed $^{b}$} & \\
\hline B3LYP & & & & Ne-matrix & & $\mathrm{N}_{2}$-matrix & \\
\hline$v$ & $\Delta v$ & $v$ & $\Delta v$ & $v$ & $\Delta v$ & $v$ & \\
\hline $3253(1)$ & 0 & $3275(9)$ & 0 & & & & $v(\mathrm{CH})$ \\
\hline $3241(1)$ & 0 & $3257(<1)$ & 0 & & & & $v(\mathrm{CH})$ \\
\hline $3211(3)$ & 0 & $3224(<1)$ & 0 & & & & $v(\mathrm{CH})$ \\
\hline $1550(13)$ & 1.6 & $1541(6)$ & 0.6 & & & & $v(\mathrm{C}=\mathrm{C})$ \\
\hline $1447(6)$ & 1.5 & $1448(25)$ & 5.2 & $1398.4 \mathrm{w}$ & 3.7 & $1396.6 \mathrm{w}$ & $v(\mathrm{C}=\mathrm{C})$ \\
\hline $1403(47)$ & 5.2 & $1404(7)$ & 0.2 & & & & $v(\mathrm{C}-\mathrm{C})+\rho(\mathrm{CH})$ \\
\hline 1368 (157) & 0 & $1369(214)$ & 0.6 & $1365.5 \mathrm{~s}$ & 0.8 & $1368.8 \mathrm{~s}$ & $v_{\text {antisym }}\left(\mathrm{SO}_{2}\right)$ \\
\hline $1321(152)$ & 22.3 & $1316(202)$ & 15.8 & $1295.5 \mathrm{~s}$ & 13.3 & $1297.0 \mathrm{~s}$ & $v(\mathrm{~N}=\mathrm{S})+\rho(\mathrm{CH})$ \\
\hline $1253(147)$ & 2.5 & $1243(22)$ & 0 & $1221.3 \mathrm{w}$ & $<0.5$ & $1244.1 \mathrm{w}$ & $\rho(\mathrm{CH})$ \\
\hline $1177(38)$ & 2.6 & $1183(62)$ & 2.3 & $1167.0 \mathrm{w}$ & $<0.5$ & $1169.0 \mathrm{w}$ & $v_{\text {antisym }}\left(\mathrm{SO}_{2}\right)+\rho(\mathrm{CH})$ \\
\hline $1106(4)$ & 0.3 & $1100(11)$ & 0.2 & & & $1092.1 \mathrm{w}$ & $\rho(\mathrm{CH})$ \\
\hline $1074(12)$ & 0.9 & $1037(14)$ & 7.8 & & & & $v(\mathrm{C}-\mathrm{C})+v(\mathrm{~N}=\mathrm{S})$ \\
\hline $968(29)$ & 8.7 & $914(11)$ & 3.3 & $905.2 \mathrm{vw}$ & $<0.5$ & $906.0 \mathrm{vw}$ & ring distortion \\
\hline $930(<1)$ & 0 & 909 (1) & 0 & & & & $\omega(\mathrm{CH})$ \\
\hline $860(31)$ & 0.2 & $891(27)$ & 1.8 & $885.1 \mathrm{w}$ & $<0.5$ & $884.8 \mathrm{w}$ & $v(\mathrm{CS})$ \\
\hline $753(3)$ & 2.3 & $833(3)$ & 1.7 & & & & $v(\mathrm{CS})$ \\
\hline 740 (14) & 8.9 & $812(66)$ & 0.3 & $785.5 \mathrm{~m}$ & $<0.5$ & $786.4 \mathrm{~m}$ & $\omega(\mathrm{CH})$ \\
\hline $712(72)$ & 0 & $698(8)$ & 0 & & & & $\omega(\mathrm{CH})$ \\
\hline $596(7)$ & 5.0 & $675(11)$ & 6.6 & & & & ring distortion \\
\hline $579(<1)$ & 0 & $640(15)$ & 0.5 & & & & $\omega(\mathrm{CH})$ \\
\hline $526(30)$ & 1.9 & $612(4)$ & 6.3 & & & & ring breathing \\
\hline $514(1)$ & 1.2 & $537(28)$ & 1.0 & & & & $\delta\left(\mathrm{SO}_{2}\right)$ \\
\hline
\end{tabular}

${ }^{a}$ Band positions and intensities: s, strong; m, medium strong; w, weak. ${ }^{b}$ Calculated harmonic IR frequencies $(v$, unscaled), ${ }^{15} \mathrm{~N}$-isotopic shifts $(\Delta v)$, and intensities $\left(\mathrm{km} \mathrm{mol}^{-1}\right.$, in parentheses) at the B3LYP/6-311++G(3df,3pd) level. ${ }^{c}$ Tentative assignment based on the calculated vibrational displacement vectors of $\mathbf{9}, v=$ stretching; $\rho=$ rocking; $\tau=$ twisting; $\omega=$ wagging; $\delta=$ bending. 
Calculated Atomic Coordinates (in Angstroms) and Energies (in Hatrees) for All Opitimized Structures.

2-thienylsulfonyl azide

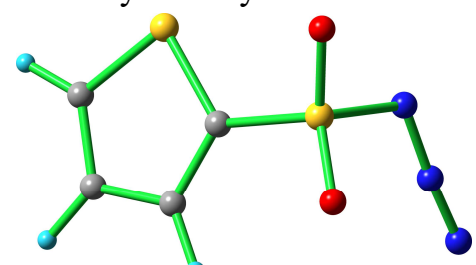

B3LYP/6-311++G(3df,3pd)

$\begin{array}{lccc}\mathrm{C} & 2.97369700 & 0.52192600 & -0.31368600 \\ \mathrm{C} & 2.45699400 & 1.33722200 & 0.65625900 \\ \mathrm{C} & 1.12434700 & 1.00190200 & 0.98925500 \\ \mathrm{C} & 0.66837900 & -0.06680000 & 0.26079300 \\ \mathrm{~S} & 1.85910800 & -0.66836500 & -0.83367800 \\ \mathrm{H} & 3.96173500 & 0.54958000 & -0.74275900 \\ \mathrm{H} & 3.01053800 & 2.13930100 & 1.11960100 \\ \mathrm{H} & 0.52620600 & 1.50174500 & 1.73492200 \\ \mathrm{~S} & -0.92540900 & -0.76666300 & 0.36652500 \\ \mathrm{O} & -0.85611800 & -2.14659100 & 0.01521400 \\ \mathrm{O} & -1.53968500 & -0.29777300 & 1.57304900 \\ \mathrm{~N} & -1.75980300 & -0.06705800 & -0.97035300 \\ \mathrm{~N} & -2.22137400 & 1.06262000 & -0.76647000 \\ \mathrm{~N} & -2.67764200 & 2.08432800 & -0.67734100\end{array}$

Zero-point correction=

0.080626

0.090550

Thermal correction to Energy=

0.091494

Thermal correction to Enthalpy=

0.043203

Thermal correction to Gibbs Free Energy=

Sum of electronic and zero-point Energies $=$

Sum of electronic and thermal Energies=

$-1265.354474$

$-1265.344550$

$-1265.343606$

Sum of electronic and thermal Free Energies=

$-1265.391897$

\section{CBS-QB3}

$\begin{array}{lrrc}\mathrm{C} & 2.98567800 & 0.52154300 & -0.32181500 \\ \mathrm{C} & 2.47442600 & 1.33587300 & 0.65524000 \\ \mathrm{C} & 1.13814800 & 1.00493700 & 0.99319400 \\ \mathrm{C} & 0.67312900 & -0.05912700 & 0.25957900 \\ \mathrm{~S} & 1.86014900 & -0.66892600 & -0.84487600 \\ \mathrm{H} & 3.97279000 & 0.54474100 & -0.75868400 \\ \mathrm{H} & 3.03426100 & 2.13578000 & 1.12003800 \\ \mathrm{H} & 0.54351300 & 1.50369000 & 1.74544600 \\ \mathrm{~S} & -0.92564800 & -0.76336500 & 0.38035600 \\ \mathrm{O} & -0.85454400 & -2.15413200 & 0.04963800 \\ \mathrm{O} & -1.54238400 & -0.25604500 & 1.57711500 \\ \mathrm{~N} & -1.75877600 & -0.08089500 & -0.99181100 \\ \mathrm{~N} & -2.23733100 & 1.04499200 & -0.78001800 \\ \mathrm{~N} & -2.71181300 & 2.06369100 & -0.68612900\end{array}$

Temperature $=298.150000$

$\mathrm{E}(\mathrm{ZPE})=0.079191$

$\mathrm{E}(\mathrm{SCF})=-1261.385317$

$\mathrm{DE}(\mathrm{CBS})=-0.206015$

$\mathrm{DE}(\mathrm{CCSD})=-0.072254$

$\mathrm{DE}($ Empirical $)=-0.087759$
Pressure $=1.000000$

$\mathrm{E}($ Thermal $)=0.089324$

$\mathrm{DE}(\mathrm{MP} 2)=-2.064174$

$\mathrm{DE}(\mathrm{MP} 34)=-0.019851$

$\mathrm{DE}(\operatorname{Int})=0.067842$ 
CBS-QB3 $(0 \mathrm{~K})=-1263.688337$

CBS-QB3 Enthalpy $=-1263.677260$

2-thienylsulfonyl nitrene (singlet)

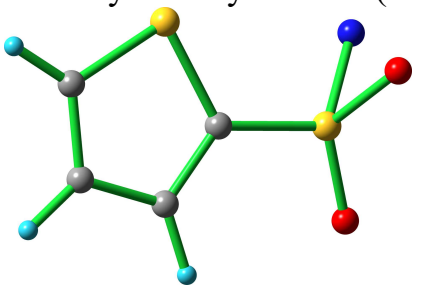

B3LYP/6-311++G(3df,3pd)

$\begin{array}{lr}\mathrm{C} & 2.70234500 \\ \mathrm{C} & 2.46641500 \\ \mathrm{C} & 1.08880300 \\ \mathrm{C} & 0.31463600 \\ \mathrm{~S} & 1.26502600 \\ \mathrm{H} & 3.65566000 \\ \mathrm{H} & 3.24828600 \\ \mathrm{H} & 0.66896800 \\ \mathrm{~S} & -1.41351900 \\ \mathrm{O} & -1.94248600 \\ \mathrm{O} & -1.88148600 \\ \mathrm{~N} & -2.00549300\end{array}$

$$
\begin{array}{cc}
-0.28735500 & 0.01754000 \\
1.06093000 & 0.07858200 \\
1.36635400 & 0.05358600 \\
0.23401200 & -0.02030100 \\
-1.20769500 & -0.07628600 \\
-0.78994800 & 0.01748300 \\
1.80224700 & 0.13604900 \\
2.35914600 & 0.08749200 \\
0.18726400 & -0.07392400 \\
-0.74914100 & 1.00139000 \\
1.53229400 & -0.17800700 \\
-1.07905900 & -0.74302400
\end{array}
$$

Zero-point correction $=$

Thermal correction to Energy=

Thermal correction to Enthalpy=

Thermal correction to Gibbs Free Energy=

Sum of electronic and zero-point Energies=

Sum of electronic and thermal Energies=

Sum of electronic and thermal Enthalpies=

Sum of electronic and thermal Free Energies=

$$
\begin{gathered}
0.069636 \\
0.077655 \\
0.078599 \\
0.035778 \\
-1155.747253 \\
-1155.739234 \\
-1155.738290 \\
-1155.781112
\end{gathered}
$$

\section{CBS-QB3}

$\begin{array}{lrrc}\mathrm{C} & 2.70975200 & -0.28693500 & 0.01557400 \\ \mathrm{C} & 2.47341000 & 1.06364300 & 0.07534800 \\ \mathrm{C} & 1.09235100 & 1.37177700 & 0.05379000 \\ \mathrm{C} & 0.31542200 & 0.23881000 & -0.01713100 \\ \mathrm{~S} & 1.26521700 & -1.21151900 & -0.07487700 \\ \mathrm{H} & 3.66304800 & -0.79418300 & 0.01362600 \\ \mathrm{H} & 3.25759200 & 1.80599500 & 0.13087000 \\ \mathrm{H} & 0.67273200 & 2.36708600 & 0.08741100 \\ \mathrm{~S} & -1.42108800 & 0.18607900 & -0.07176100 \\ \mathrm{O} & -1.93925200 & -0.75676900 & 1.01563100 \\ \mathrm{O} & -1.88816800 & 1.53816000 & -0.17673900 \\ \mathrm{~N} & -2.00367100 & -1.07810800 & -0.76604700\end{array}$

Temperature $=298.150000$

Pressure $=1.000000$ $\mathrm{E}($ Thermal $)=0.076541$ $\mathrm{DE}(\mathrm{MP} 2)=-1.658083$

$\mathrm{E}(\mathrm{SCF})=-1152.377980$

$\mathrm{DE}(\mathrm{CBS})=-0.169245$

$\mathrm{DE}(\mathrm{CCSD})=-0.061646$

$\mathrm{DE}($ Empirical $)=-0.071988$

CBS-QB3 $(0 \mathrm{~K})=-1154.249711$

CBS-QB3 Enthalpy= -1154.240568

$\mathrm{DE}(\mathrm{MP} 34)=-0.035292$

$\mathrm{DE}(\mathrm{Int})=0.056180$

CBS-QB3 Energy $=-1154.241512$

CBS-QB3 Free Energy= -1154.283764

2-thienylsulfonyl nitrene (triplet) 


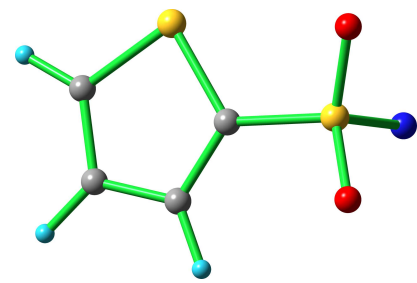

B3LYP/6-311++G(3df,3pd)

$\begin{array}{lrrr}\mathrm{C} & -2.69455800 & -0.16740100 & 0.02179200 \\ \mathrm{C} & -2.36576800 & 1.16595000 & 0.00477900 \\ \mathrm{C} & -0.97414000 & 1.37396800 & -0.03507900 \\ \mathrm{C} & -0.27531000 & 0.18651800 & -0.05872500 \\ \mathrm{~S} & -1.32509300 & -1.18591700 & -0.01771700 \\ \mathrm{H} & -3.68156700 & -0.59985300 & 0.04183600 \\ \mathrm{H} & -3.09647300 & 1.95987000 & 0.01369800 \\ \mathrm{H} & -0.48835100 & 2.33693900 & -0.05875600 \\ \mathrm{~S} & 1.45511200 & 0.02039400 & -0.06248200 \\ \mathrm{O} & 1.80608500 & -1.27461100 & -0.57822400 \\ \mathrm{O} & 2.04058400 & 1.22286400 & -0.58781600 \\ \mathrm{~N} & 1.75305500 & 0.00159700 & 1.57402000\end{array}$

Zero-point correction=

0.068915

0.077130

Thermal correction to Energy=

0.078074

Thermal correction to Enthalpy=

0.033844

Thermal correction to Gibbs Free Energy=

Sum of electronic and zero-point Energies=

Sum of electronic and thermal Energies=

$-1155.772556$

$-1155.764341$

$-1155.763397$

thalpies $=$

$-1155.807628$

\section{CBS-QB3}

$\begin{array}{ll}\mathrm{C} & -2.70298700 \\ \mathrm{C} & -2.37191500 \\ \mathrm{C} & -0.97657000 \\ \mathrm{C} & -0.27633300 \\ \mathrm{~S} & -1.32805000 \\ \mathrm{H} & -3.69071100 \\ \mathrm{H} & -3.10414000 \\ \mathrm{H} & -0.48865800 \\ \mathrm{~S} & 1.46177900 \\ \mathrm{O} & 1.80569900 \\ \mathrm{O} & 2.04301600 \\ \mathrm{~N} & 1.76013400\end{array}$

Temperature $=298.150000$

$\mathrm{E}(\mathrm{ZPE})=0.067784$

$\mathrm{E}(\mathrm{SCF})=-1152.467110$

$\mathrm{DE}(\mathrm{CBS})=-0.163737$

$\mathrm{DE}(\mathrm{CCSD})=-0.061744$

$\mathrm{DE}($ Empirical $)=-0.071468$

CBS-QB3 $(0 \mathrm{~K})=-1154.263955$

CBS-QB3 Enthalpy= -1154.254665

$$
\begin{array}{cc}
-0.16579200 & 0.02140700 \\
1.16947800 & 0.00421800 \\
1.37828100 & -0.03471800 \\
0.18944200 & -0.05454000 \\
-1.19040600 & -0.01675400 \\
-0.60204700 & 0.04092400 \\
1.96510100 & 0.01231200 \\
2.34257400 & -0.06074400 \\
0.01987700 & -0.06385500 \\
-1.28215100 & -0.58489600 \\
1.23244100 & -0.58847300 \\
-0.00113700 & 1.58085700 \\
\text { Pressure }=1.000000 \\
\text { E(Thermal })=0.076129 \\
\text { DE(MP2) }=-1.566999 \\
\text { DE(MP34) }=-0.053189 \\
\text { DE(Int) }=0.052508
\end{array}
$$

CBS-QB3 Energy= -1154.255609

CBS-QB3 Free Energy= -1154.299143

2-thienyl- $N$-sulfonylamine 


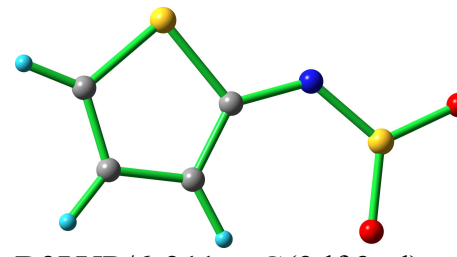

B3LYP/6-311++G(3df,3pd)

$\begin{array}{lccc}\mathrm{C} & -3.01972900 & 0.13796900 & -0.00007600 \\ \mathrm{C} & -2.36749700 & 1.33876000 & 0.00000900 \\ \mathrm{C} & -0.95860900 & 1.20667100 & 0.00007100 \\ \mathrm{C} & -0.56062100 & -0.10908300 & 0.00007800 \\ \mathrm{~S} & -1.92784600 & -1.18360300 & -0.00003100 \\ \mathrm{H} & -4.08150000 & -0.04119200 & -0.00013600 \\ \mathrm{H} & -2.87729400 & 2.29020900 & -0.00009500 \\ \mathrm{H} & -0.26649000 & 2.03274500 & 0.00011000 \\ \mathrm{~N} & 0.68479800 & -0.72263300 & 0.00021200 \\ \mathrm{~S} & 2.01425100 & -0.01689300 & -0.00003200 \\ \mathrm{O} & 2.13196300 & 1.41773400 & -0.00003900 \\ \mathrm{O} & 3.17903000 & -0.85039400 & -0.00006500\end{array}$

Zero-point correction $=$

0.070640

0.078690

Thermal correction to Energy=

0.079634

Thermal correction to Enthalpy=

0.036273

Thermal correction to Gibbs Free Energy=

Sum of electronic and zero-point Energies

Sum of electronic and thermal Energies=

$-1155.852875$

$-1155.844825$

$-1155.843881$

$-1155.887241$

Sum of electronic and thermal Free Energies=

CBS-QB3

$\begin{array}{lccc}\mathrm{C} & -3.02432700 & 0.14176800 & 0.00001000 \\ \mathrm{C} & -2.36824200 & 1.34293000 & 0.00002100 \\ \mathrm{C} & -0.95627500 & 1.20889300 & -0.00004000 \\ \mathrm{C} & -0.55747200 & -0.10949200 & -0.00008100 \\ \mathrm{~S} & -1.93051600 & -1.18862500 & 0.00009100 \\ \mathrm{H} & -4.08792100 & -0.03978400 & 0.00000300 \\ \mathrm{H} & -2.87728400 & 2.29740200 & 0.00002700 \\ \mathrm{H} & -0.26174200 & 2.03570800 & -0.00008900 \\ \mathrm{~N} & 0.68580700 & -0.73566900 & -0.00015100 \\ \mathrm{~S} & 2.01664400 & -0.01575200 & -0.00028900 \\ \mathrm{O} & 2.12085300 & 1.42794200 & 0.00028300 \\ \mathrm{O} & 3.18991400 & -0.85021800 & 0.00031900\end{array}$

Temperature $=298.150000$

$\mathrm{E}(\mathrm{ZPE})=0.069554$

$\mathrm{E}(\mathrm{SCF})=-1152.482465$

$\mathrm{DE}(\mathrm{CBS})=-0.169128$

$\mathrm{DE}(\mathrm{CCSD})=-0.059222$

$\mathrm{DE}($ Empirical $)=-0.072215$

CBS-QB3 $(0 \mathrm{~K})=-1154.345349$

CBS-QB3 Enthalpy $=-1154.336261$

3-thienylsulfonyl azide
Pressure $=1.000000$

$\mathrm{E}($ Thermal $)=0.077698$

$\mathrm{DE}(\mathrm{MP} 2)=-1.665124$

$\mathrm{DE}(\mathrm{MP} 34)=-0.022906$

$\mathrm{DE}(\mathrm{Int})=0.056158$

CBS-QB3 Energy $=-1154.337205$

CBS-QB3 Free Energy $=-1154.379839$ 


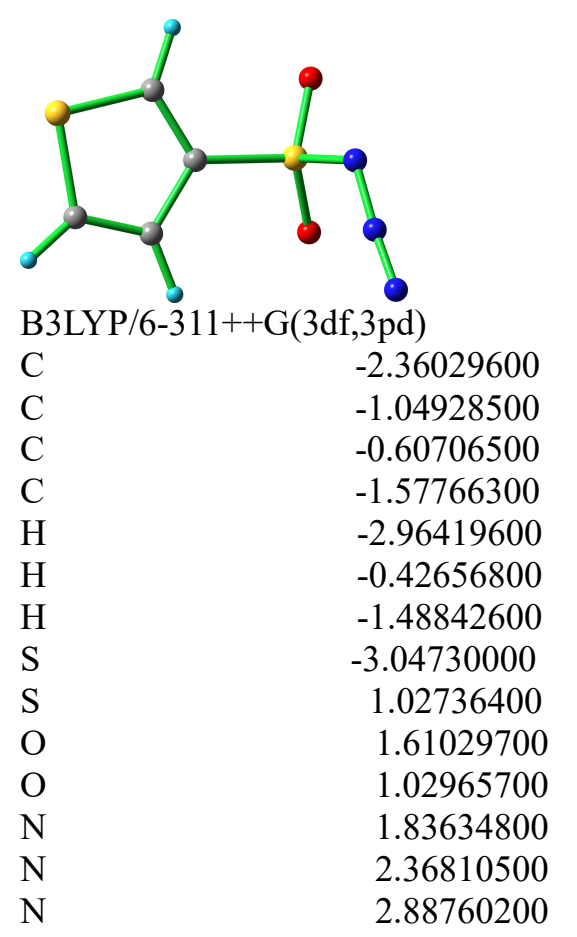

Zero-point correction $=$

Thermal correction to Energy=

Thermal correction to Enthalpy=

Thermal correction to Gibbs Free Energy=

Sum of electronic and zero-point Energies=

Sum of electronic and thermal Energies=

Sum of electronic and thermal Enthalpies=

Sum of electronic and thermal Free Energies=

\section{CBS-QB3}

$\begin{array}{lc}\mathrm{C} & -2.37483200 \\ \mathrm{C} & -1.06219500 \\ \mathrm{C} & -0.61507800 \\ \mathrm{C} & -1.58105800 \\ \mathrm{H} & -2.98672800 \\ \mathrm{H} & -0.44032800 \\ \mathrm{H} & -1.48996100 \\ \mathrm{~S} & -3.05973700 \\ \mathrm{~S} & 1.02538300 \\ \mathrm{O} & 1.61211900 \\ \mathrm{O} & 1.02534300 \\ \mathrm{~N} & 1.82894000 \\ \mathrm{~N} & 2.39356600 \\ \mathrm{~N} & 2.94406100\end{array}$

Temperature $=298.150000$

$\mathrm{E}(\mathrm{ZPE})=0.079083$

$\mathrm{E}(\mathrm{SCF})=-1261.388876$

$\mathrm{DE}(\mathrm{CBS})=-0.205636$

$\mathrm{DE}(\mathrm{CCSD})=-0.071926$

$\mathrm{DE}($ Empirical $)=-0.087797$

CBS-QB3 $(0 \mathrm{~K})=-1263.690268$

CBS-QB3 Enthalpy= -1263.679220

3-thienylsulfonyl nitrene (singlet)

$\begin{array}{rc}1.24988000 & 0.60021500 \\ 0.98949600 & 0.85829800 \\ -0.17765100 & 0.17320300 \\ -0.77626300 & -0.58219500 \\ 2.05381600 & 0.98549100 \\ 1.57579300 & 1.51490200 \\ -1.66767000 & -1.17842200 \\ 0.08287800 & -0.46260800 \\ -0.80731100 & 0.26964500 \\ -0.38943000 & 1.51128700 \\ -2.16582300 & -0.16584900 \\ 0.02665300 & -1.00819500 \\ 1.09564000 & -0.68576300 \\ 2.07174400 & -0.49106800\end{array}$

0.080506

0.090415

0.091359

0.043064

$-1265.357134$

$-1265.347225$

$-1265.346281$

$-1265.394575$

$1.25355900 \quad 0.60231400$

$0.99356800 \quad 0.86449100$

$-0.17135800 \quad 0.17269400$

$-0.77439800 \quad-0.58881100$

$2.05466000 \quad 0.98686200$

$1.57762000 \quad 1.52734600$

$-1.66592700-1.18823600$

$0.08448700 \quad-0.47307800$

$\begin{array}{ll}-0.80367600 & 0.28234800\end{array}$

$-0.34748700 \quad 1.51582500$

$-2.17402000-0.13407700$

$0.01948200-1.03395500$

$1.07319300 \quad-0.70149400$

$2.03653800-0.49775300$

Pressure $=1.000000$

$\mathrm{E}($ Thermal $)=0.089186$

$\mathrm{DE}(\mathrm{MP} 2)=-2.062317$

$\mathrm{DE}(\mathrm{MP} 34)=-0.020514$

$\mathrm{DE}(\operatorname{Int})=0.067717$

CBS-QB3 Energy= -1263.680164

CBS-QB3 Free Energy= -1263.727965 


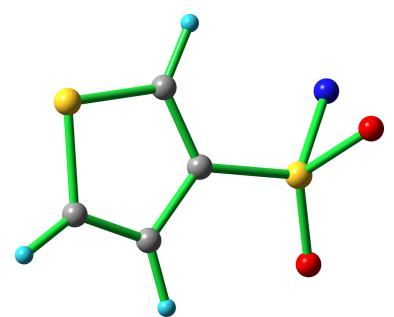

B3LYP/6-311++G(3df,3pd)

$\begin{array}{lrrr}\mathrm{C} & 2.30555000 & 1.04984900 & 0.11030100 \\ \mathrm{C} & 0.97366700 & 1.32797300 & 0.09293700 \\ \mathrm{C} & 0.19796500 & 0.14043300 & -0.03128100 \\ \mathrm{C} & 0.94708300 & -1.00471600 & -0.11614600 \\ \mathrm{H} & 3.12666900 & 1.74118000 & 0.19449200 \\ \mathrm{H} & 0.54850400 & 2.31586700 & 0.16270900 \\ \mathrm{H} & 0.58826600 & -2.01354600 & -0.22611700 \\ \mathrm{~S} & 2.60787400 & -0.64021700 & -0.03134500 \\ \mathrm{~S} & -1.54518200 & 0.11389500 & -0.08464000 \\ \mathrm{O} & -2.08767000 & -0.77422300 & 1.02712700 \\ \mathrm{O} & -1.99942900 & 1.45964500 & -0.24103000 \\ \mathrm{~N} & -2.15933000 & -1.16956400 & -0.69985100\end{array}$

Zero-point correction $=$

Thermal correction to Energy=

0.069480

0.077483

0.078427

Thermal correction to Enthalpy=

0.035588

Sum of electronic and zero-point Energies=

$-1155.749438$

$-1155.741434$

$-1155.740490$

$-1155.783329$

Sum of electronic and thermal Enthalpies=

Sum of electronic and thermal Free Energies=

$-1155.783329$

CBS-QB3

$\begin{array}{lrrr}\mathrm{C} & 2.30878900 & 1.05578700 & 0.11418400 \\ \mathrm{C} & 0.97408100 & 1.33193700 & 0.10076300 \\ \mathrm{C} & 0.19865500 & 0.14065600 & -0.02531500 \\ \mathrm{C} & 0.94706400 & -1.00658600 & -0.11789600 \\ \mathrm{H} & 3.13376200 & 1.74575300 & 0.19973600 \\ \mathrm{H} & 0.54582500 & 2.32071800 & 0.17351200 \\ \mathrm{H} & 0.59015200 & -2.01739300 & -0.23535100 \\ \mathrm{~S} & 2.61576700 & -0.64112700 & -0.03527500 \\ \mathrm{~S} & -1.55304300 & 0.11139600 & -0.08417100 \\ \mathrm{O} & -2.09053300 & -0.76746400 & 1.04968200 \\ \mathrm{O} & -2.00430300 & 1.46224500 & -0.26202400 \\ \mathrm{~N} & -2.15516600 & -1.18034300 & -0.70834700\end{array}$

Temperature $=298.150000$

Pressure $=1.000000$

$\mathrm{E}(\mathrm{ZPE})=0.068164$

$\mathrm{E}($ Thermal $)=0.076346$

$\mathrm{E}(\mathrm{SCF})=-1152.380676$

$\mathrm{DE}(\mathrm{CBS})=-0.169081$

$\mathrm{DE}(\mathrm{MP} 2)=-1.657047$

$\mathrm{DE}(\mathrm{MP} 34)=-0.035648$

$\mathrm{DE}(\mathrm{Int})=0.056135$

$\mathrm{DE}($ Empirical $)=-0.072018$

CBS-QB3 $(0 \mathrm{~K})=-1154.251365$

CBS-QB3 Enthalpy $=-1154.242238$

CBS-QB3 Energy $=-1154.243182$

CBS-QB3 Free Energy= -1154.285439

3-thienylsulfonyl nitrene (triplet) 


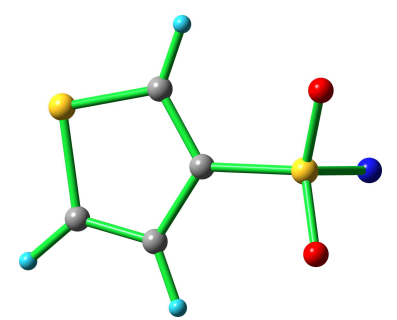

B3LYP/6-311++G(3df,3pd)

$\begin{array}{lccc}\mathrm{C} & -2.22074100 & 1.11934600 & 0.00791800 \\ \mathrm{C} & -0.87689800 & 1.32133600 & -0.01767600 \\ \mathrm{C} & -0.16440500 & 0.08665100 & -0.04560500 \\ \mathrm{C} & -0.97911300 & -1.01916300 & -0.03004000 \\ \mathrm{H} & -3.00351600 & 1.85871800 & 0.02085400 \\ \mathrm{H} & -0.40041600 & 2.28834500 & -0.03324200 \\ \mathrm{H} & -0.67796600 & -2.05236700 & -0.05201500 \\ \mathrm{~S} & -2.61726900 & -0.56004900 & 0.00261500 \\ \mathrm{~S} & 1.57887000 & -0.04066200 & -0.06669400 \\ \mathrm{O} & 1.96027600 & -1.34965400 & -0.52512700 \\ \mathrm{O} & 2.14052500 & 1.14786100 & -0.64916000 \\ \mathrm{~N} & 1.90525800 & 0.01171400 & 1.57091200\end{array}$

Zero-point correction $=$

0.068819

0.077014

0.077958

Thermal correction to Enthalpy=

Thermal correction to Gibbs Free Energy=

0.033714

$-1155.774243$

$-1155.766048$

$-1155.765103$

$-1155.809348$

Sum of electronic and thermal Enthalpies=

Sum of electronic and thermal Free Energies=

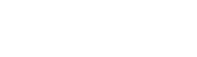

CBS-QB3

C $\quad-2.22409200$

C -0.87745800

$1.12508900 \quad 0.00798200$

$1.32530100-0.01613600$

C $\quad-0.16564200$

C $\quad-0.97915400$

$\begin{array}{rr}0.08679400 & -0.04039900\end{array}$

$-1.02203700 \quad-0.02881100$

$\begin{array}{llll}\mathrm{H} & -3.01090600 & 1.86327700 & 0.01952000\end{array}$

$\begin{array}{llll}\mathrm{H} & -0.39754500 & 2.29301400 & -0.03276100\end{array}$

$\begin{array}{llll}\mathrm{H} & -0.67962500 & -2.05779400 & -0.05327200\end{array}$

$\begin{array}{llll}\mathrm{S} & -2.62512500 & -0.56160100 & 0.00207500\end{array}$

$\begin{array}{llll}\mathrm{S} & 1.58545800 & -0.04170000 & -0.06829400\end{array}$

$\begin{array}{llll}\mathrm{O} & 1.95946000 & -1.36102600 & -0.52328600\end{array}$

$\begin{array}{llll}\mathrm{O} & 2.14182200 & 1.15319100 & -0.65857100\end{array}$

$\begin{array}{llll}\mathrm{N} & 1.91293800 & 0.01801900 & 1.57786300\end{array}$

Temperature $=298.150000$

$\mathrm{E}(\mathrm{ZPE})=0.067647$

$\mathrm{E}(\mathrm{SCF})=-1152.470400$

$\mathrm{DE}(\mathrm{CBS})=-0.163757$

$\mathrm{DE}(\mathrm{CCSD})=-0.059515$

$\mathrm{DE}($ Empirical $)=-0.071012$

CBS-QB3 $(0 \mathrm{~K})=-1154.265886$

CBS-QB3 Enthalpy $=-1154.256608$

Pressure $=1.000000$

$\mathrm{E}($ Thermal $)=0.075980$

$\mathrm{DE}(\mathrm{MP} 2)=-1.569878$

$\mathrm{DE}(\mathrm{MP} 34)=-0.051763$

$\mathrm{DE}(\mathrm{Int})=0.052792$

CBS-QB3 Energy $=-1154.257552$

CBS-QB3 Free Energy $=-1154.301127$

3-thienyl- $N$-sulfonylamine 


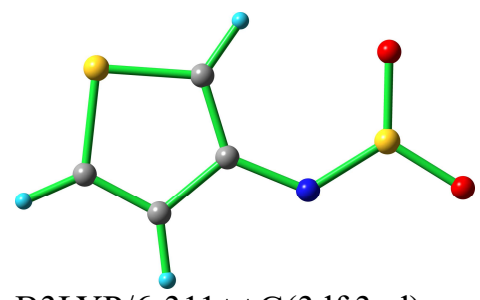

B3LYP/6-311++G(3df,3pd)

$\begin{array}{lccc}\mathrm{C} & -2.73097500 & 0.91732200 & 0.00027700 \\ \mathrm{C} & -1.47990000 & 1.44905000 & 0.00026500 \\ \mathrm{C} & -0.46356700 & 0.44547300 & -0.00004900 \\ \mathrm{C} & -0.97836100 & -0.82949400 & -0.00033600 \\ \mathrm{H} & -3.67359900 & 1.43712900 & 0.00045000 \\ \mathrm{H} & -1.25917400 & 2.50491700 & 0.00045200 \\ \mathrm{H} & -0.44119000 & -1.76070300 & -0.00052000 \\ \mathrm{~S} & -2.68793400 & -0.80159300 & -0.00015100 \\ \mathrm{~N} & 0.87733600 & 0.86563100 & -0.00024000 \\ \mathrm{~S} & 2.08323800 & -0.03071600 & 0.00005900 \\ \mathrm{O} & 1.99192300 & -1.46744100 & 0.00057900 \\ \mathrm{O} & 3.36114600 & 0.61519900 & -0.00035000\end{array}$

Zero-point correction $=$

Thermal correction to Energy=

0.070717

0.078683

0.079627

Thermal correction to Enthalpy=

0.036465

$-1155.855069$

$-1155.847103$

$-1155.846159$

$-1155.889321$

Sum of electronic and thermal Enthalpies=

Sum of electronic and thermal Free Energies=

\section{CBS-QB3}

$\mathrm{C}$

C

$\mathrm{C}$

C

$\mathrm{H}$

$\mathrm{H}$

$\mathrm{H}$

$\mathrm{S}$

$\mathrm{N}$

$\mathrm{S}$

$\mathrm{O}$

$\mathrm{O}$

$$
\begin{gathered}
-2.73380700 \\
-1.48067400 \\
-0.46037700 \\
-0.97359300 \\
-3.68060900 \\
-1.25726100 \\
-0.43689500 \\
-2.69074900 \\
0.88121300 \\
2.08461700 \\
1.97719500 \\
3.37219000
\end{gathered}
$$

Temperature $=298.150000$

$\mathrm{E}(\mathrm{ZPE})=0.069668$

$\mathrm{E}(\mathrm{SCF})=-1152.487115$

$\mathrm{DE}(\mathrm{CBS})=-0.168951$

$\mathrm{DE}(\mathrm{CCSD})=-0.058880$

$\mathrm{DE}($ Empirical $)=-0.072280$

CBS-QB3 $(0 \mathrm{~K})=-1154.348499$

CBS-QB3 Enthalpy= 1154.339507

3-thienylnitrene (singlet)

$$
\begin{array}{cc}
0.92235400 & 0.00017500 \\
1.45380800 & 0.00018100 \\
0.44901000 & 0.00001800 \\
-0.82947100 & -0.00018100 \\
1.43896700 & 0.00028300 \\
2.51133300 & 0.00029500 \\
-1.76322000 & -0.00036100 \\
-0.80494300 & 0.00003700 \\
0.88032600 & -0.00002000 \\
-0.03251300 & -0.00003900 \\
-1.47607100 & -0.00007300 \\
0.61053600 & -0.00007700
\end{array}
$$

Pressure $=1.000000$

$\mathrm{E}($ Thermal $)=0.077716$

$\mathrm{DE}(\mathrm{MP} 2)=-1.663353$

$\mathrm{DE}(\mathrm{MP} 34)=-0.023682$

$\mathrm{DE}(\mathrm{Int})=0.056095$

CBS-QB3 Energy $=-1154.340451$

CBS-QB3 Free Energy= 1154.382793 


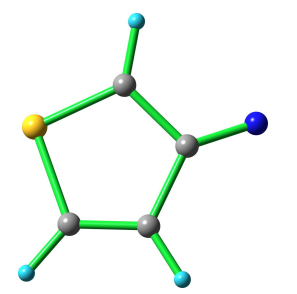

$\begin{array}{lrcc}\text { B3LYP/6-311++G(3df,3pd) } & & \\ \text { C } & 0.85350600 & 1.18441700 & 0.00540400 \\ \text { C } & -0.47062200 & 1.32238100 & 0.09999500 \\ \text { C } & -1.17300100 & 0.01598400 & 0.13613300 \\ \text { C } & -0.18933500 & -1.05171400 & 0.26695600 \\ \text { S } & 1.36488100 & -0.51868600 & -0.10287800 \\ \text { H } & 1.60807100 & 1.94807400 & -0.08646000 \\ \text { H } & -0.99617300 & 2.26430700 & 0.11746400 \\ \text { H } & -0.44628200 & -2.09056900 & 0.38258600 \\ \text { N } & -2.30385700 & -0.37846300 & -0.25978200\end{array}$

Zero-point correction=

$$
\begin{gathered}
0.057448 \\
0.062660 \\
0.063605 \\
0.028320 \\
-607.075925 \\
-607.070712 \\
-607.069768 \\
-607.105053
\end{gathered}
$$

Sum of electronic and thermal Free Energies=

\section{CBS-QB3}

$\begin{array}{lrrc}\mathrm{C} & 0.85484100 & 1.18985400 & 0.00590500 \\ \mathrm{C} & -0.47094400 & 1.33096000 & 0.10394200 \\ \mathrm{C} & -1.17852700 & 0.02255900 & 0.14078500 \\ \mathrm{C} & -0.19583400 & -1.04926500 & 0.28438300 \\ \mathrm{~S} & 1.36646000 & -0.52467500 & -0.10804900 \\ \mathrm{H} & 1.61543400 & 1.94999500 & -0.09029800 \\ \mathrm{H} & -0.99426100 & 2.27663700 & 0.12021100 \\ \mathrm{H} & -0.45354200 & -2.08943700 & 0.40521900 \\ \mathrm{~N} & -2.29831500 & -0.38672100 & -0.27377600\end{array}$

Temperature $=298.150000$

$\mathrm{E}(\mathrm{ZPE})=0.056636$

$E(S C F)=-605.120923$

$\mathrm{DE}(\mathrm{CBS})=-0.097255$

$\mathrm{DE}(\mathrm{CCSD})=-0.052941$

$\mathrm{DE}($ Empirical $)=-0.042547$

CBS-QB3 (0 K) $=-606.235095$

CBS-QB3 Enthalpy=-606.228849

Pressure $=1.000000$

$\mathrm{E}($ Thermal $)=0.061938$

$\mathrm{DE}(\mathrm{MP} 2)=-0.973740$

$\mathrm{DE}(\mathrm{MP} 34)=-0.038855$

$\mathrm{DE}(\operatorname{Int})=0.034530$

CBS-QB3 Energy $=-606.229793$

CBS-QB3 Free Energy=-606.264337

3-thienylnitrene (triplet)

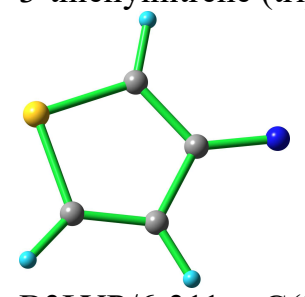

B3LYP/6-311++G(3df,3pd)
$\mathrm{C}$
$-0.82265200$
1.18058700
$-0.00031100$ 


$\begin{array}{lrrc}\mathrm{C} & 0.52462000 & 1.23357600 & 0.00030400 \\ \mathrm{C} & 1.13916400 & -0.08902400 & -0.00027800 \\ \mathrm{C} & 0.14745600 & -1.09759200 & -0.00000900 \\ \mathrm{~S} & -1.42834400 & -0.44732300 & 0.00008500 \\ \mathrm{H} & -1.51532600 & 2.00582900 & -0.00039600 \\ \mathrm{H} & 1.10557900 & 2.14224500 & 0.00066300 \\ \mathrm{H} & 0.31963900 & -2.15991900 & 0.00012900 \\ \mathrm{~N} & 2.43029800 & -0.31375200 & -0.00000100 \\ & & \\ \text { Zero-point correction= } & & 0.057659 \\ \text { Thermal correction to Energy= } & 0.062612 \\ \text { Thermal correction to Enthalpy= } & 0.063557 \\ \text { Thermal correction to Gibbs Free Energy= } & 0.028048 \\ \text { Sum of electronic and zero-point Energies }= & -607.111613 \\ \text { Sum of electronic and thermal Energies }= & -607.106660 \\ \text { Sum of electronic and thermal Enthalpies }= & -607.105715 \\ \text { Sum of electronic and thermal Free Energies }= & -607.141224\end{array}$

\section{CBS-QB3}

$\begin{array}{lrrr}\mathrm{C} & 0.82327000 & 1.18609100 & -0.00003600 \\ \mathrm{C} & -0.52667300 & 1.23552700 & 0.00015300 \\ \mathrm{C} & -1.14217900 & -0.08974700 & 0.00019200 \\ \mathrm{C} & -0.14991800 & -1.09983400 & 0.00001100 \\ \mathrm{~S} & 1.43431500 & -0.44872300 & -0.00031000 \\ \mathrm{H} & 1.51973800 & 2.01086700 & -0.00008500 \\ \mathrm{H} & -1.11040500 & 2.14505300 & 0.00029900 \\ \mathrm{H} & -0.31951800 & -2.16466200 & 0.00000600 \\ \mathrm{~N} & -2.43797900 & -0.31484600 & 0.00040300\end{array}$

Temperature $=298.150000$

Pressure $=1.000000$

$\mathrm{E}($ Thermal $)=0.061956$

$\mathrm{E}(\mathrm{ZPE})=0.056943$

$\mathrm{E}(\mathrm{SCF})=-605.219246$

$\mathrm{DE}(\mathrm{CBS})=-0.093695$

$\mathrm{DE}(\mathrm{CCSD})=-0.046499$

$\mathrm{DE}($ Empirical $)=-0.044542$

CBS-QB3 $(0 \mathrm{~K})=-606.262303$

CBS-QB3 Enthalpy $=-606.256346$

$\mathrm{DE}(\mathrm{MP} 2)=-0.887131$

$\mathrm{DE}(\mathrm{MP} 34)=-0.058874$

$\mathrm{DE}(\mathrm{Int})=0.030742$

CBS-QB3 Energy $=-606.257291$

CBS-QB3 Free Energy $=-606.291955$

4-thioxo-2-butenenitrile (anti-anti)

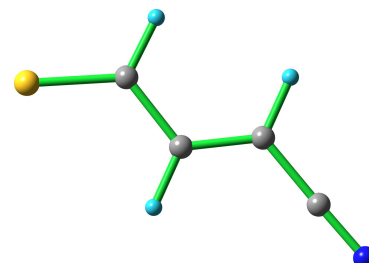

B3LYP/6-311++G(3df,3pd)

$\begin{array}{lrrr}\mathrm{C} & 1.18299200 & 0.50982600 & 0.00000800 \\ \mathrm{H} & 1.16310200 & 1.59932400 & 0.00003000 \\ \mathrm{C} & -0.10924900 & -0.13163800 & 0.00000800 \\ \mathrm{H} & -0.13266600 & -1.21358600 & 0.00002400 \\ \mathrm{C} & -1.25228900 & 0.58102200 & -0.00000600 \\ \mathrm{H} & -1.22812300 & 1.66435300 & -0.00002100 \\ \mathrm{C} & -2.53916400 & -0.01412400 & -0.00000300 \\ \mathrm{~N} & -3.59679600 & -0.47407800 & 0.00000000 \\ \mathrm{~S} & 2.60509500 & -0.27512900 & -0.00000500\end{array}$

Zero-point correction=

0.058430 
Thermal correction to Energy=

0.064579

Thermal correction to Enthalpy=

0.065524

Thermal correction to Gibbs Free Energy=

Sum of electronic and zero-point Energies=

0.027996

Sum of electronic and thermal Energies=

$-607.162659$

$-607.156509$

Sum of electronic and thermal Enthalpies=

$-607.155565$

Sum of electronic and thermal Free Energies=

$-607.193093$

4-thioxo-2-butenenitrile (anti-syn)

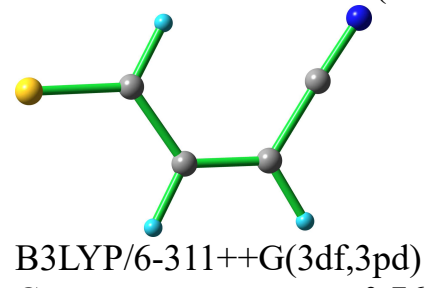

$\begin{array}{lrrr}\mathrm{C} & -0.76681100 & -0.25293000 & 0.00019100 \\ \mathrm{H} & -0.20065900 & -1.18245400 & 0.00077900 \\ \mathrm{C} & 0.01215600 & 0.96207500 & -0.00007700 \\ \mathrm{H} & -0.53663100 & 1.89468300 & -0.00029800 \\ \mathrm{C} & 1.35979500 & 1.00213200 & 0.00004800 \\ \mathrm{H} & 1.87560300 & 1.95362200 & 0.00009000 \\ \mathrm{C} & 2.19507900 & -0.14675800 & 0.00016800 \\ \mathrm{~N} & 2.90277000 & -1.05734600 & -0.00021600 \\ \mathrm{~S} & -2.39118900 & -0.29072100 & -0.00006500\end{array}$

Zero-point correction $=$

Thermal correction to Energy=

0.058606

0.064648

0.065592

Thermal correction to Enthalpy=

0.028046

Sum of electronic and zero-point Energies=

$-607.161534$

$-607.155493$

$-607.154549$

Sum of electronic and thermal Enthalpies=

$-607.192095$

Sum of electronic and thermal Free Energies=

4-thioxo-2-butenenitrile (syn-anti)

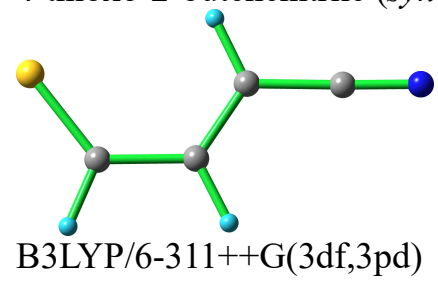

$\begin{array}{lrrr}\mathrm{C} & 1.30316000 & 0.81298500 & -0.00025900 \\ \mathrm{H} & 1.75481000 & 1.80294100 & 0.00059300 \\ \mathrm{C} & -0.15237900 & 0.82980000 & -0.00004800 \\ \mathrm{H} & -0.62544300 & 1.80415800 & 0.00026000 \\ \mathrm{C} & -0.92160500 & -0.27231000 & -0.00021100 \\ \mathrm{H} & -0.46421600 & -1.25497600 & -0.00051800 \\ \mathrm{C} & -2.33691900 & -0.22059000 & -0.00000100 \\ \mathrm{~N} & -3.49047200 & -0.20415500 & 0.00016400 \\ \mathrm{~S} & 2.27591300 & -0.48889700 & 0.00010200\end{array}$

Zero-point correction=

0.058244

Thermal correction to Energy=

0.064484

Thermal correction to Enthalpy=

0.065428

Thermal correction to Gibbs Free Energy=

0.027391

Sum of electronic and zero-point Energies=

$-607.159019$ 
Sum of electronic and thermal Energies=

$-607.152779$

Sum of electronic and thermal Enthalpies=

$-607.151835$

Sum of electronic and thermal Free Energies=

$-607.189872$

4-thioxo-2-butenenitrile (syn-syn)

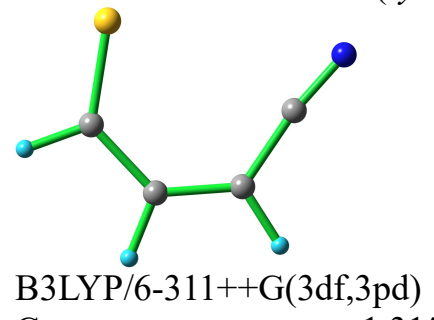

$\begin{array}{lrrr}\mathrm{C} & -1.31571600 & 0.70742200 & -0.00003900 \\ \mathrm{H} & -2.21534100 & 1.32086600 & 0.00002900 \\ \mathrm{C} & -0.09142700 & 1.48811100 & -0.00000200 \\ \mathrm{H} & -0.23286200 & 2.56257000 & 0.00001600 \\ \mathrm{C} & 1.18714400 & 1.06356600 & 0.00001300 \\ \mathrm{H} & 1.97507500 & 1.80678400 & 0.00004200 \\ \mathrm{C} & 1.66391900 & -0.27164400 & -0.00000200 \\ \mathrm{~N} & 2.19498000 & -1.29622300 & -0.00001000 \\ \mathrm{~S} & -1.47220300 & -0.90883700 & 0.00001000\end{array}$

Zero-point correction $=$

0.058320

0.064502

Thermal correction to Energy=

0.065446

Thermal correction to Enthalpy=

0.026856

Sum of electronic and zero-point Energies=

$-607.153742$

$-607.147559$

$-607.146615$

Sum of electronic and thermal Enthalpies=

$-607.185206$

Sum of electronic and thermal Free Energies=

thienyl radical

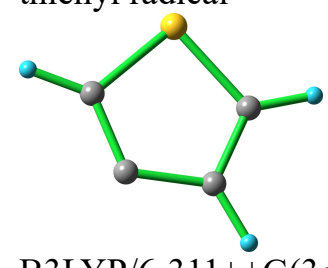

B3LYP/6-311++G(3df,3pd)

$\begin{array}{lrrc}\mathrm{C} & -0.44030600 & -1.12172100 & 0.00008300 \\ \mathrm{C} & -1.48354700 & -0.23618500 & -0.00004600 \\ \mathrm{C} & -0.98322200 & 1.08060300 & -0.00001900 \\ \mathrm{C} & 0.34958900 & 1.24906000 & 0.00006600 \\ \mathrm{~S} & 1.08768400 & -0.32852800 & -0.00003900 \\ \mathrm{H} & -0.48541500 & -2.19846600 & 0.00013000 \\ \mathrm{H} & -2.52494400 & -0.51546200 & -0.00008700 \\ \mathrm{H} & 0.95232500 & 2.13982900 & 0.00007600\end{array}$

Zero-point correction $=$

0.053809

Thermal correction to Energy=

0.057881

Thermal correction to Enthalpy=

0.058825

Thermal correction to Gibbs Free Energy=

0.025997

Sum of electronic and zero-point Energies=

$-552.347349$

Sum of electronic and thermal Energies=

$-552.343277$

$-552.342333$

Sum of electronic and thermal Enthalpies $=$

$-552.375160$ 
$1.08059200-0.00003600$

$1.25545200 \quad 0.00003900$

$\begin{array}{ll}-0.32553400 & -0.00000800\end{array}$

$-2.20638700 \quad 0.00009700$

$-0.52708200$

$-0.00008300$

Temperature $=298.150000$

2.14862000

0.00005600

$\mathrm{E}(\mathrm{ZPE})=0.053131$

$E(S C F)=-550.722334$

$\mathrm{DE}(\mathrm{CBS})=-0.077217$

$\mathrm{DE}(\mathrm{CCSD})=-0.042231$

$\mathrm{DE}($ Empirical $)=-0.037083$

CBS-QB3 $(0 \mathrm{~K})=-551.585679$

CBS-QB3 Enthalpy=-551.580612

Pressure $=1.000000$
$E($ Thermal) $=0.057254$
DE(MP2) $=-0.740682$
DE(MP34) $=-0.045594$
DE(Int) $=0.026330$

CBS-QB3 Energy $=-551.581556$

CBS-QB3 Free Energy $=-551.613523$

Bicyclic azirine- I

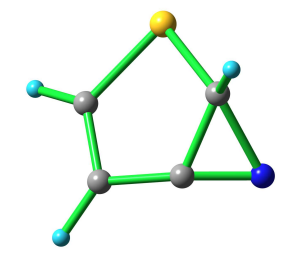

B3LYP/6-311++G(3df,3pd)

$\begin{array}{lrrc}\mathrm{C} & 0.94498600 & 1.05309000 & -0.04671100 \\ \mathrm{C} & -0.30736200 & 1.52911500 & 0.15087900 \\ \mathrm{C} & -1.15403400 & 0.36380800 & 0.17647400 \\ \mathrm{C} & -0.57860000 & -0.93170400 & 0.40950300 \\ \mathrm{~S} & 1.14206000 & -0.69756600 & -0.09287700 \\ \mathrm{H} & 1.79741700 & 1.66736800 & -0.29868600 \\ \mathrm{H} & -0.60192500 & 2.56455200 & 0.13123700 \\ \mathrm{H} & -0.77863200 & -1.57585900 & 1.25218400 \\ \mathrm{~N} & -1.73139500 & -0.51155200 & -0.53422400\end{array}$

Zero-point correction=

0.058547

Thermal correction to Energy=

0.063325

Thermal correction to Enthalpy=

0.064270

Thermal correction to Gibbs Free Energy=

Sum of electronic and zero-point Energies=

Sum of electronic and thermal Energies=

0.030263

$-607.087910$

$-607.083132$

Sum of electronic and thermal Enthalpies=

$-607.082188$

Sum of electronic and thermal Free Energies=

$-607.116195$

Bicyclic azirine- II

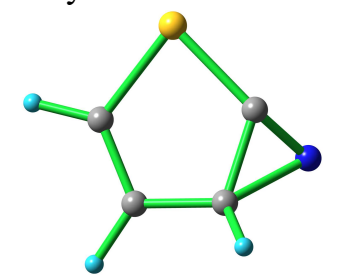

B3LYP/6-311++G(3df,3pd)
$\mathrm{C}$
0.77139000
1.13925500
$-0.19395000$
C
$-0.54365500$
1.41060800
$-0.08366400$ 


$\begin{array}{lrrc}\mathrm{C} & -1.32548300 & 0.25296500 & 0.39181200 \\ \mathrm{C} & -0.43685200 & -0.86696300 & 0.21129700 \\ \mathrm{~S} & 1.28554700 & -0.55130100 & 0.08271000 \\ \mathrm{H} & 1.55975900 & 1.84772200 & -0.39890000 \\ \mathrm{H} & -0.93189100 & 2.41459700 & -0.17974300 \\ \mathrm{~N} & -1.41556700 & -1.05403700 & -0.55107300 \\ \mathrm{H} & -2.08005000 & 0.32156700 & 1.15982400 \\ & & \\ \text { Zero-point correction = } & 0.058501 \\ \text { Thermal correction to Energy= } & 0.063393 \\ \text { Thermal correction to Enthalpy= } & 0.064337 \\ \text { Thermal correction to Gibbs Free Energy= } & 0.030109 \\ \text { Sum of electronic and zero-point Energies }= & -607.081279 \\ \text { Sum of electronic and thermal Energies }= & -607.076388 \\ \text { Sum of electronic and thermal Enthalpies }= & -607.075443 \\ \text { Sum of electronic and thermal Free Energies }= & -607.109671\end{array}$

